\title{
Creating, Using and Updating Thesauri Files For AutoMap and ORA
}

\author{
Abhinav Sangal, Kathleen M. Carley, \\ Neal Altman, Michael K. Martin
}

July 26, 2012

CMU-ISR-12-108

Institute for Software Research

School of Computer Science

Carnegie Mellon University

Pittsburgh, PA 15213

Contact Information:

\{sangala\}@andrew.cmu.edu,

\{carley, na22, mkmartin\}@cs.cmu.edu

Center for the Computational Analysis of Social and Organizational Systems

CASOS technical report.

This work was supported in part by the Office of Naval Research - ONR -N000140811223. (SORASCS), ONR -000140910667 (CATNET), ONR - N000140811186 (Ethnographic). Additional support was provided by the center for Computational Analysis of Social and Organizational Systems (CASOS). The views and conclusions contained in this document are those of the authors and should not be interpreted as representing the official policies, either expressed or implied, of the Office of Naval Research, the Department of Defense or the U.S. government. 
Keywords: Automap, ORA, Thesaurus, Universal Thesaurus, Meta-ontology, Domain Thesaurus, Split, Merge, Algorithm. 


\begin{abstract}
AutoMap [1] is text analysis software that performs Network Text Analysis by running an automated process on a corpus of raw text data to generate one or more meta-networks which include the nodes and links representing relations among entities described. Automap uses thesaurus files [1] when creating meta-networks. These thesaurus files are list which allows the association of words or phrases found in texts with abstract concepts and/or node classes used in the extracted meta-networks.
\end{abstract}

Over time, a large number of thesauri have been created. Many of the extant thesauri contain entries that are relevant to new text analysis projects. But thesaurus re-use is difficult due to the number of thesauri. In this report, we describe one approach to making thesaurus re-use easier by combining and reconciling multiple thesauri into one under user control.

With this approach, the process of creating a Meta network out of a raw corpus of text data is more efficient and the user is able to perform a more accurate analysis of the Meta network, as the individual thesauri files can be merged to create a single and large Universal or Master Thesaurus containing all the general abstract concepts, along with several different Domainspecific thesauri.

In the following report, we first discuss the differences between a Universal thesaurus and the domain or the project specific thesauri. We then go on to discuss the evolution in the formats of the thesauri used by AutoMap, followed by a discussion of the standard Dynamic Network Analysis (DNA) meta-ontology [1].

We then detail the process used to create a single universal/master thesaurus and several different Domain thesauri. The process involves a mix of two major processes which we refer to as the Split routine and the Merge routine. We shall discuss the Split routine and the merge routine algorithm along with the process that has been used to merge and create a single thesaurus file by combining a large number of thesauri files. The merge process is not a simple process of combining all the files into one file; it involves some computational functions to make this process more efficient and more accurate. These functions are deleting duplicates, detecting the concept cycles and performing a depth first search for each concept.

The paper concludes by discussing some future improvements which could be made to the process so as to improve and automate the process which is being used at present for the merge and split process. 


\section{Table of Contents}

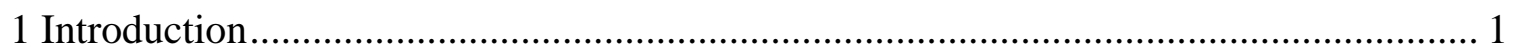

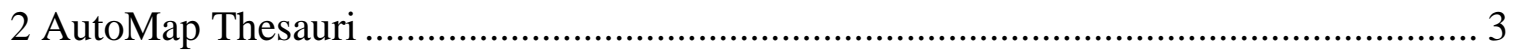

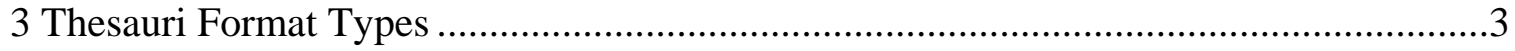

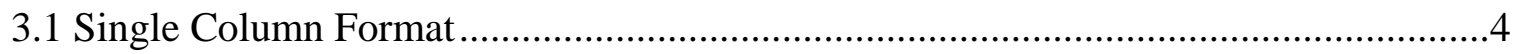

3.2 Two Column Generalization Format .......................................................................

3.3 Two Column Meta Network Format................................................................6

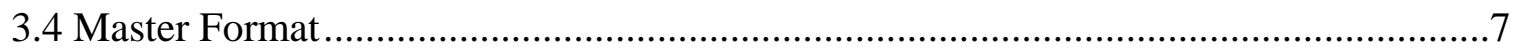

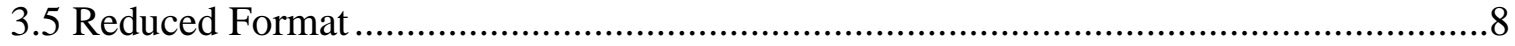

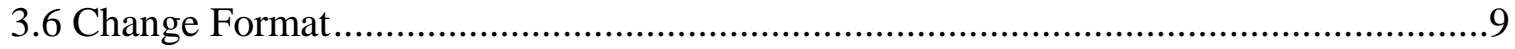

3 Evolution of the AutoMap Thesaurus..................................................................... 10

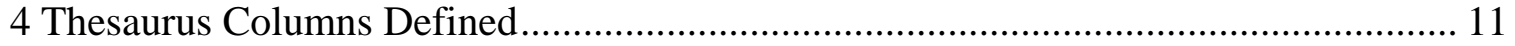

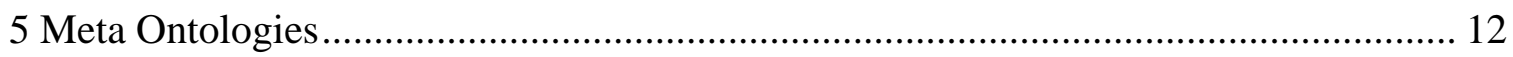

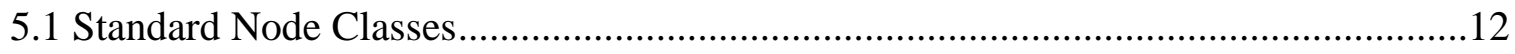

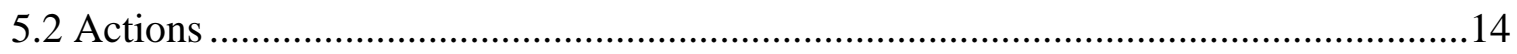

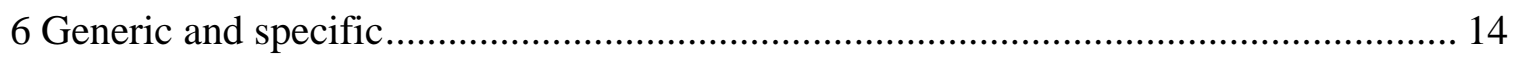

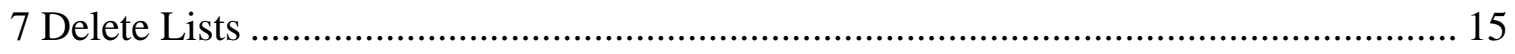

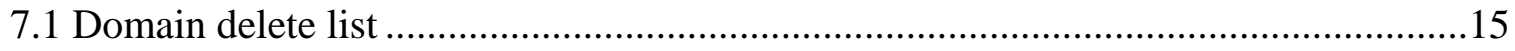

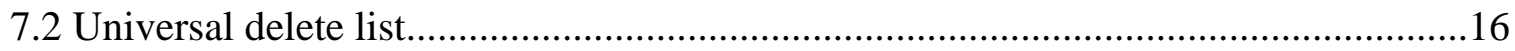

8 Difference between the Domain Thesauri and the Universal Thesaurus...................... 17

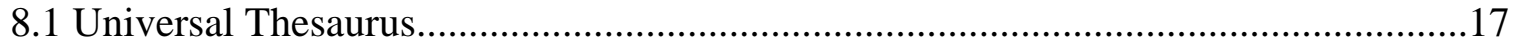

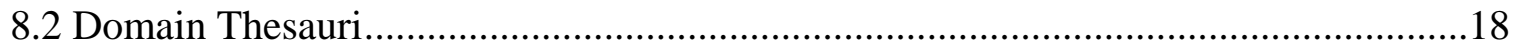

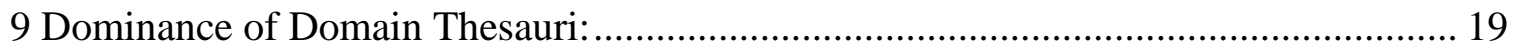

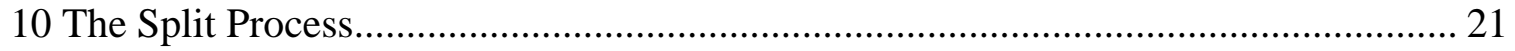

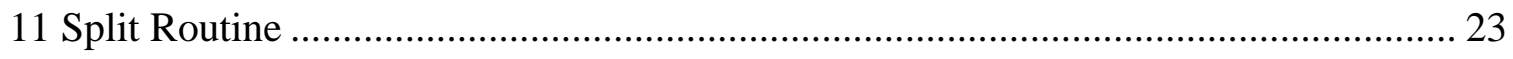

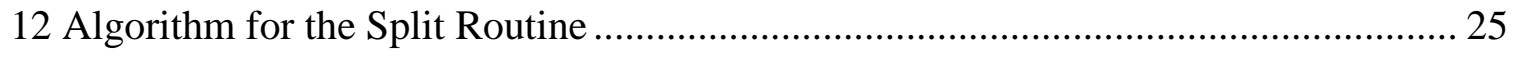


13 The Merge Process.................................................................................................. 26

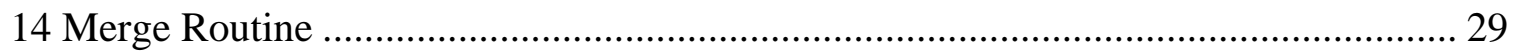

15 Algorithm for the Merge Routine ...................................................................... 31

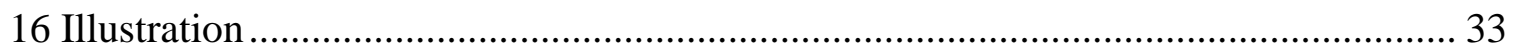

17 Creating and Applying Change Files in ORA ……………………………….......... 39

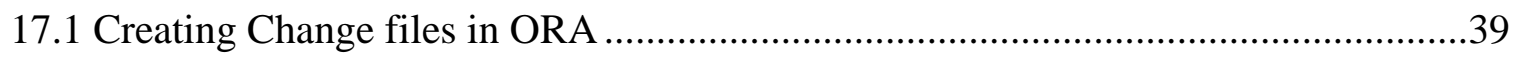

17.2 Applying change files in ORA...............................................................................45

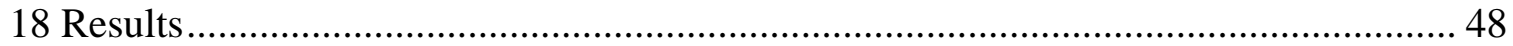

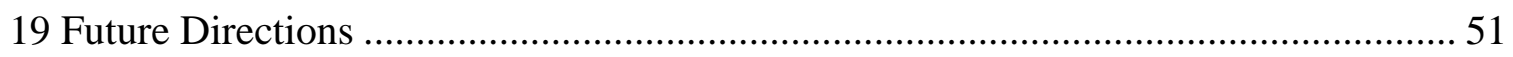

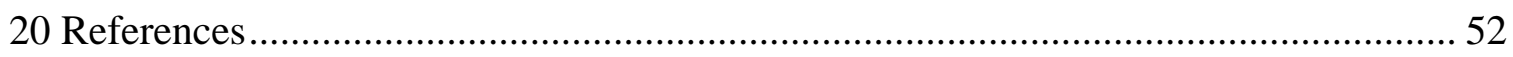




\section{Introduction}

AutoMap [1] is software tool for computer-assisted Network Text Analysis (NTA). NTA encodes the links among concepts in a text and constructs a network of the links among concepts. AutoMap subsumes classical Content Analysis by analyzing the existence, frequencies, and covariance of terms and themes.

For the purpose of NTA and in order to generate a Meta network from a corpus of raw data, AutoMap uses some files for reference. The files are referred to as the Thesaurus files. Thesaurus files are essentially lists of words in comma separated values (.csv) format. The thesaurus files are used for many purposes during the text analysis process. They can be used to create a delete list, can contain a list of noise words for filtering and even project specific concepts.

Thesauri files are an integral part of the network analysis procedure. Over time, many thesauri have been developed. As more thesauri are created, managing them has become progressively more difficult. To improve and polish the process of network analysis, we need to create a better and more efficient thesaurus.

In order to create a more efficient thesaurus, one approach is to merge all the existing files. But this is not sufficient. We have to merge the files in a way that no entry is duplicated and no concept cycles are formed. Concept cycles are the relational cycles which are formed while performing the merge operation on the thesauri files. For example: $\mathrm{A}$ maps to $\mathrm{B}$ and then $\mathrm{B}$ maps to $C$ and then we find that $C$ maps to $A$, so we say that this is a concept cycle and we just map A to $\mathrm{A}$ in the thesaurus then. For specific bodies of text, we thought that it would be useful to create supplemental domain thesauri files separate from the universal thesaurus because these domain thesauri contain entries that are not universally relevant. The major difference between the Universal thesaurus and the domain thesauri is that the universal thesauri contains general abstract concepts which may be useful in almost every project relating to generate a network from the data set. In contrast, the domain thesauri can be visualized as the project specific thesauri which contain concepts which are specific to the project. Hence, to enhance the efficiency of creating the Meta network, we incorporate a Split routine along with the merge process.

The approach for differentiating the universal thesaurus from the domain thesaurus can vary from person to person. In this report we differentiate the domain and the universal thesaurus by distinguishing the concepts which are single word agents from the others. For example, consider a data set on American politics. Now the concept entry, Obama shall go to the domain thesauri since this concept is a one word agent without any white space. Whereas, the concept Barack Obama should go the universal thesaurus since it is a two-word agent containing white spaces 
The following section starts by discussing the functions of thesauri and the types into which it can be classified according to the varying file formats, functions and according to the type of concept entries it contains. We discuss the types of thesauri based on the format and how the evolution took place with regard to the formats of the thesauri files. We continue by explaining what each of the columns contain and their meaning. Each concept in the data set can be classified into a semantic category (i.e., a node class representing a particular type of entity) according to the context in which it is used in the texts; this could be agent, organization, location, resource, event etc. The aggregation of these categories is known as the meta ontology. We also briefly discuss the various meta ontologies and how each concept can be classified into the correct ontology or can be deleted and ignored.

After discussing the format and purpose of the thesaurus, we discuss the difference between the universal thesaurus and the domain thesauri. We also discuss the precedence of the domain thesauri over the universal thesaurus and how this can be used in AutoMap during meta-network extraction. Next, we discuss the split and the merge routines and the simplified process used for carrying out the merge and split process. Also, we discuss the algorithm for the split routine and the merge routine in a very simple computing linguistics.

Finally, we conclude the report by discussing the benefits and some problems which the present split and merge process provides. Also, we discuss the various other future improvements which should be made to this process in order to achieve an efficient universal thesaurus and hence a relevant Meta network from the data set. 


\section{AutoMap Thesauri}

A thesaurus is a list which associates actions or abstract concepts with words or phrases found in text. Apart from this, a thesaurus can be used to provide additional information about the listed concepts. A thesaurus can be created by AutoMap or manually with a text editor as a text file (.txt) or a spreadsheet program that can save as (.csv) files.

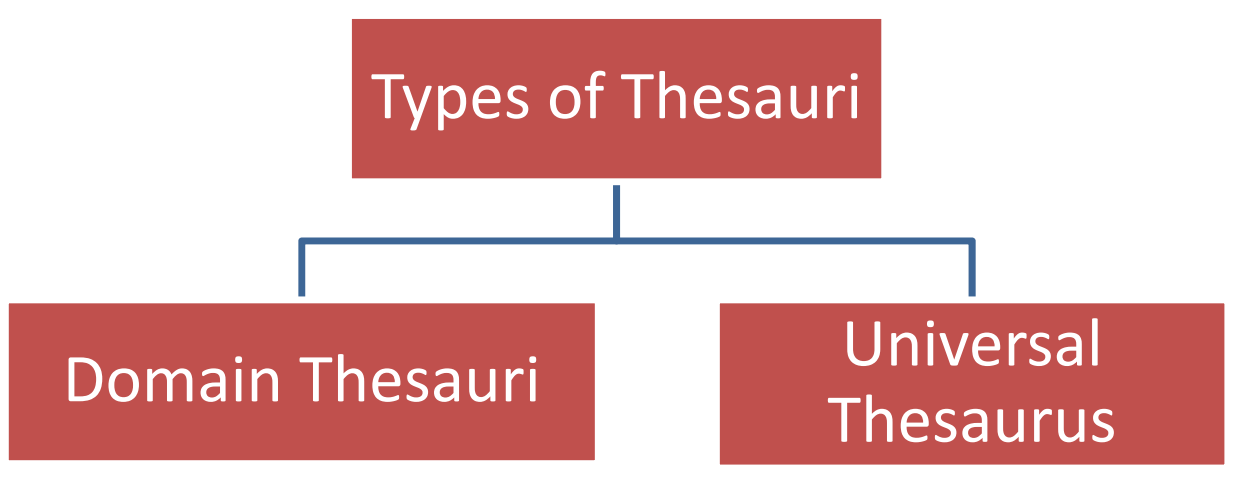

Figure 1 Major types of thesauri.

\section{Thesauri Format Types}

Thesauri can be classified into six types on the basis of the format of the thesaurus, these include the single column format, 2 column generalization format, 2 column meta network | format, reduced format, change_or review format. A thesaurus file can be in one of six types:

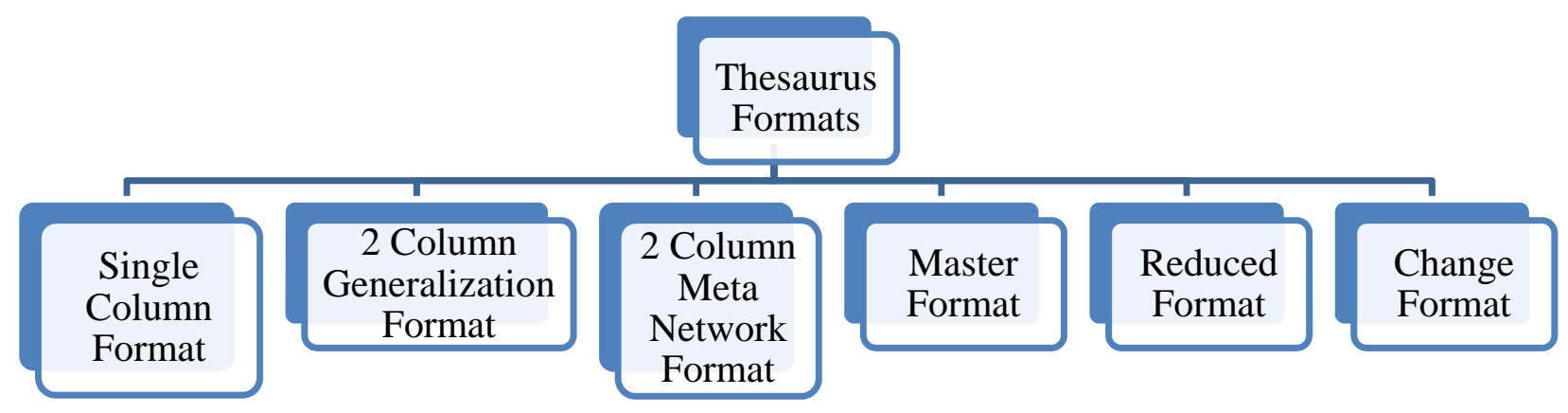

Fig 2: Thesaurus formats 


\subsection{Single Column Format}

The single column format is the least complex of the thesaurus formats. It consists of only one column, without a column header. The single column format is primarily used for delete lists. Delete lists are lists which when used as input thesauri to AutoMap, delete any of the concepts listed from the data set so they will not be included while generating the Meta network.

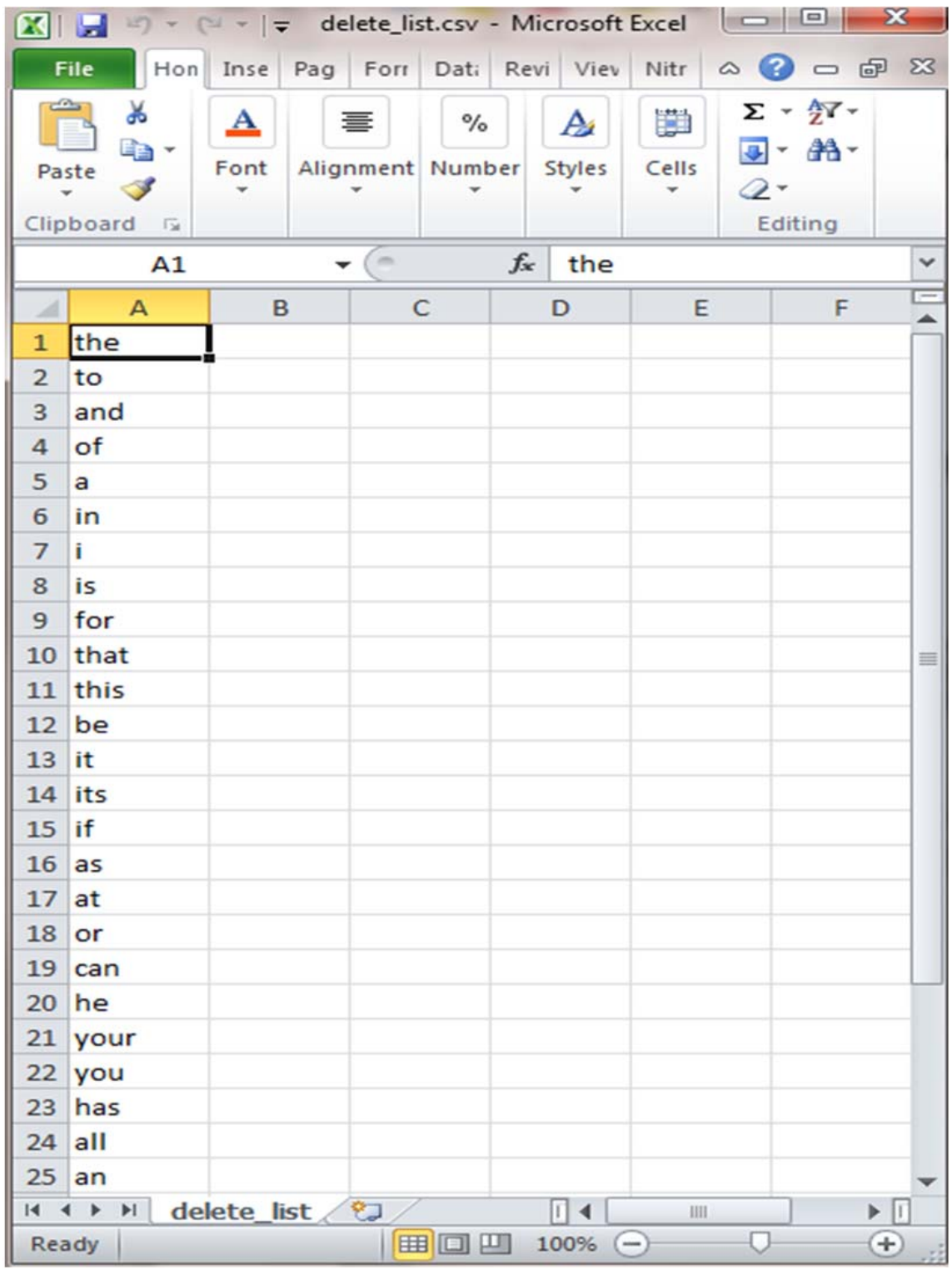

Fig 3: Single column format type. 


\subsection{Two Column Generalization Format}

Typically a two-column thesaurus is used to associate text-level concepts with higher-level concepts, where the text-level concepts represents the content of a data set, and the higher level concepts represent the text-level concepts in a generalized way. No column headers are included.

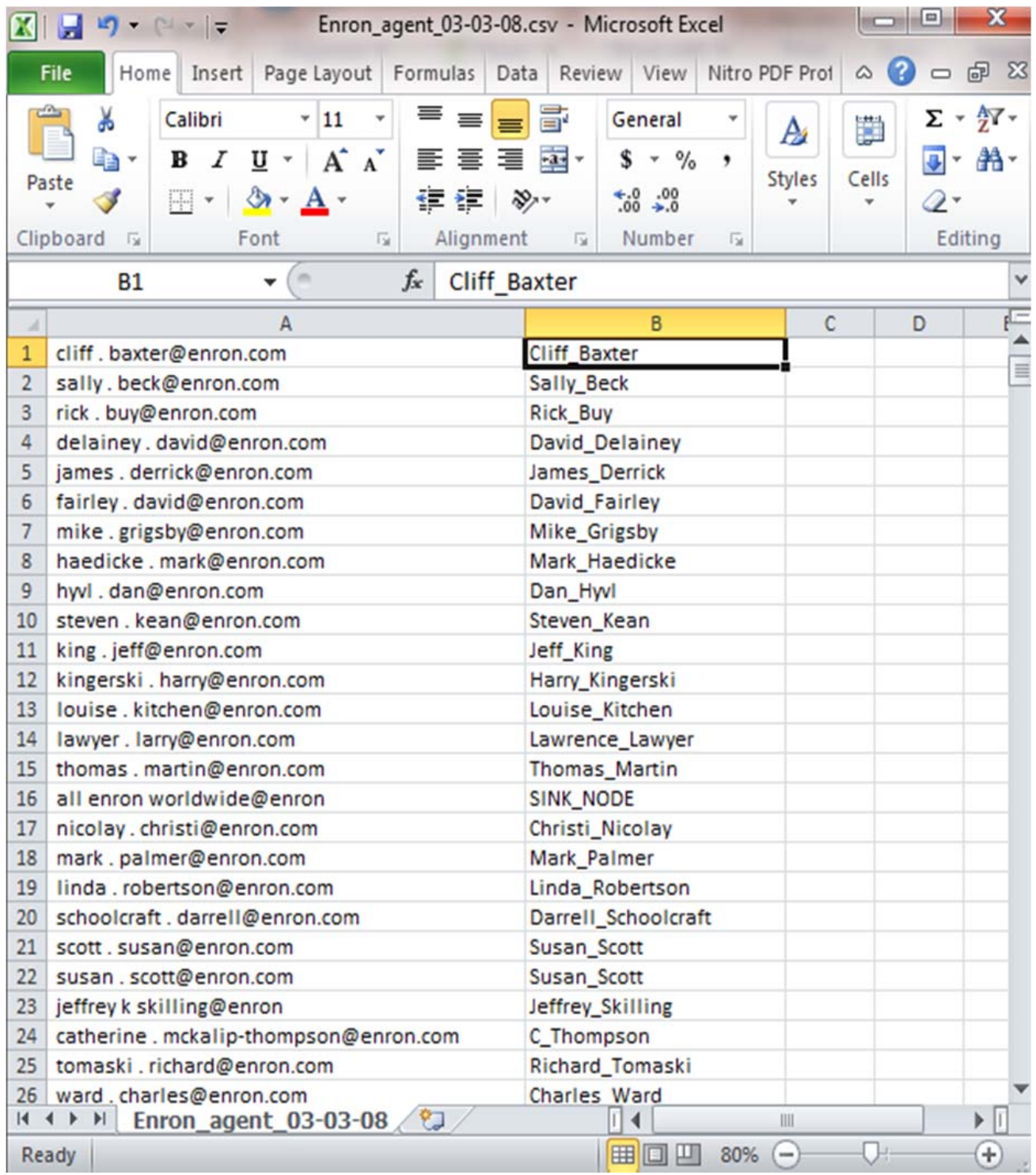

Fig 4: The two column generalization format type. 


\subsection{Two Column Meta Network Format}

This is a variation of the basic two column thesaurus which has the concept in one column and the meta ontology (see section 5 Meta Ontologies ) in the other one. Associates text level concepts with the Meta network category (i.e., node class).

This differs from the two column generalization thesaurus in the sense that the second column is the meta ontology rather than the conceptTo column in generalization thesaurus. Note however the regular two columns and meta network format can only be determined by inspection of the second column or by file naming conventions.

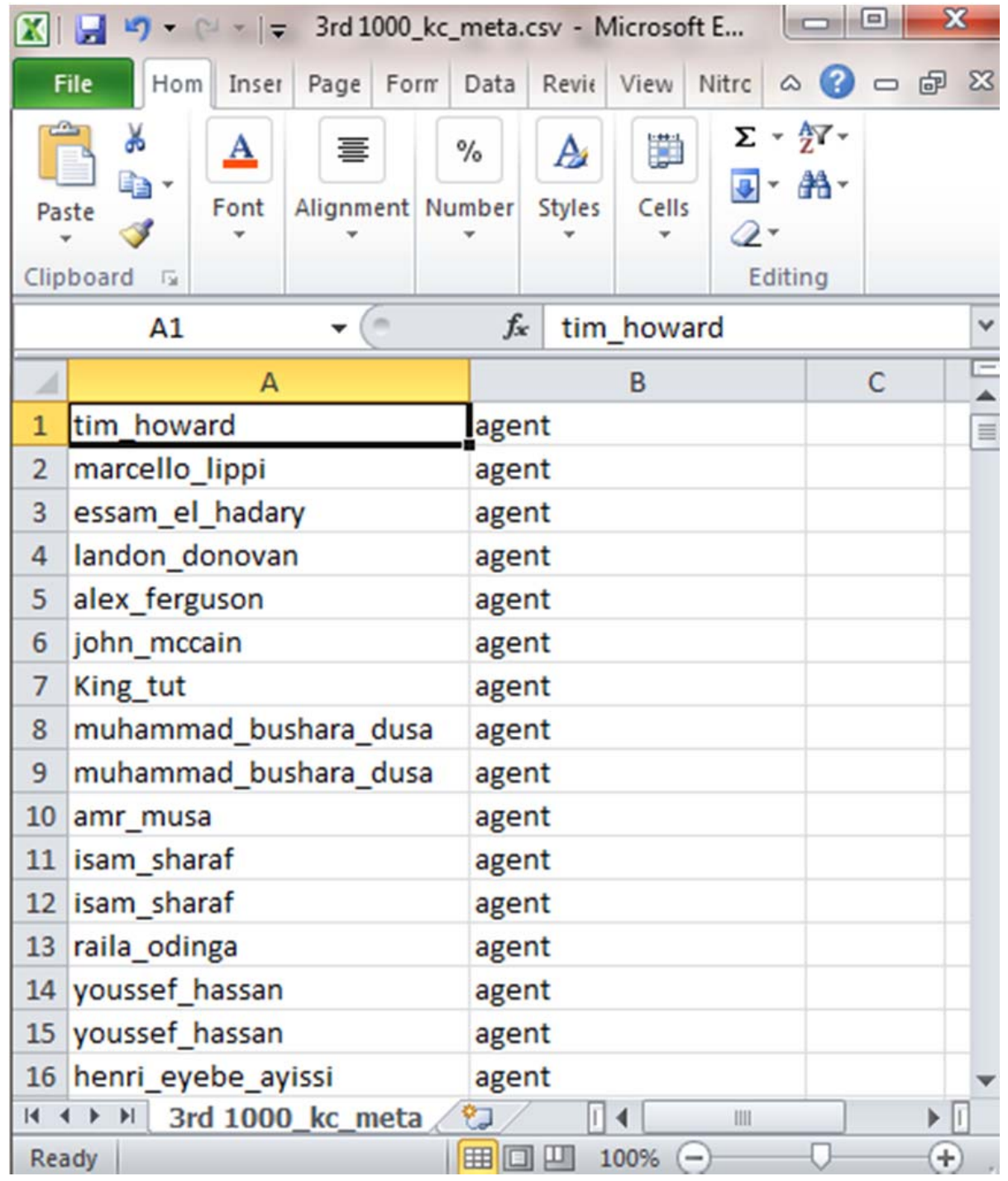

Fig 5: Two column Meta network format type. 


\subsection{Master Format}

The master format contains the same information as the two-column format plus additional attribute information. It associates the text-level concepts with higher-level concepts, where the text-level concepts refer to the content of a data-set and the higher level concepts represent the text-level concepts in a generalized way. In addition the, master thesauri format contains metaOntology column which describes the node class to which a particular concept can be categorized (for example, Barack Obama maps to agent and India maps to location). The metaName column defines whether the concept from the text is a generic concept or a specific one (see section 6 Generic and specific). The master thesaurus format thus combines the two column formats with additional information.

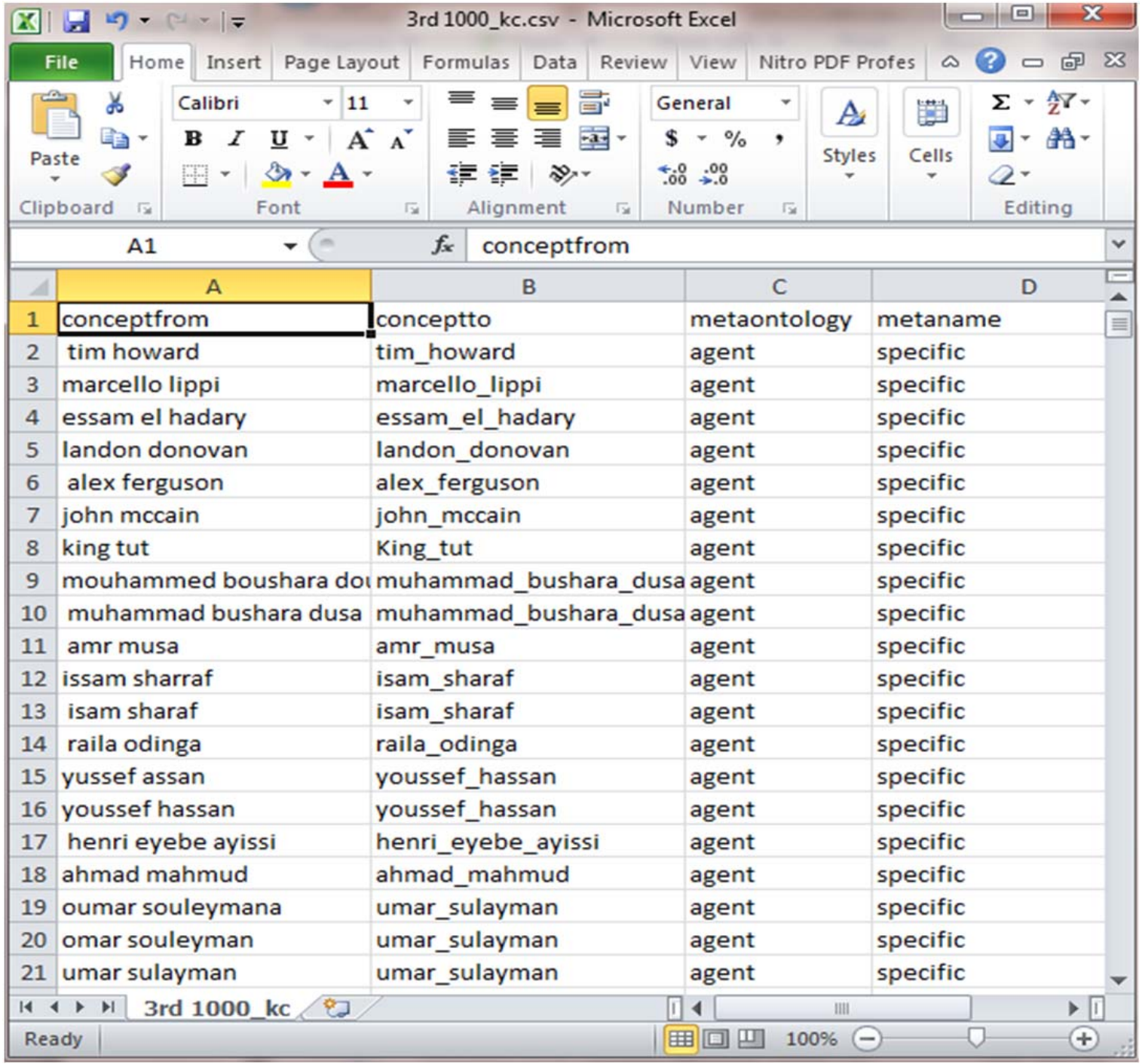

Fig 6: Master thesauri format. 


\subsection{Reduced Format}

This format subsumes the previous 3 formats with respect to the data it contains. There are 7 columns: Frequency, cuurent_concept, new_concept, current_metaontology, new_metaontology, current_metatype, new_metatype. This format provides users with a means to change metanetwork structure (i.e., nodes and/or links) by deleting, generalizing, or re-classifying nodes (i.e., entities extracted from text). The meta-type attribute value may also be changed. For example: If a concept USA has been classified as location and the user wants this concept to be an Organization instead, the user can change the meta-ontology by putting the new meta ontology in the new_metaontology column (see sections 5 Meta Ontologies on page 12 and 6 Generic and specific on page 14). This format is also known as the Review format.

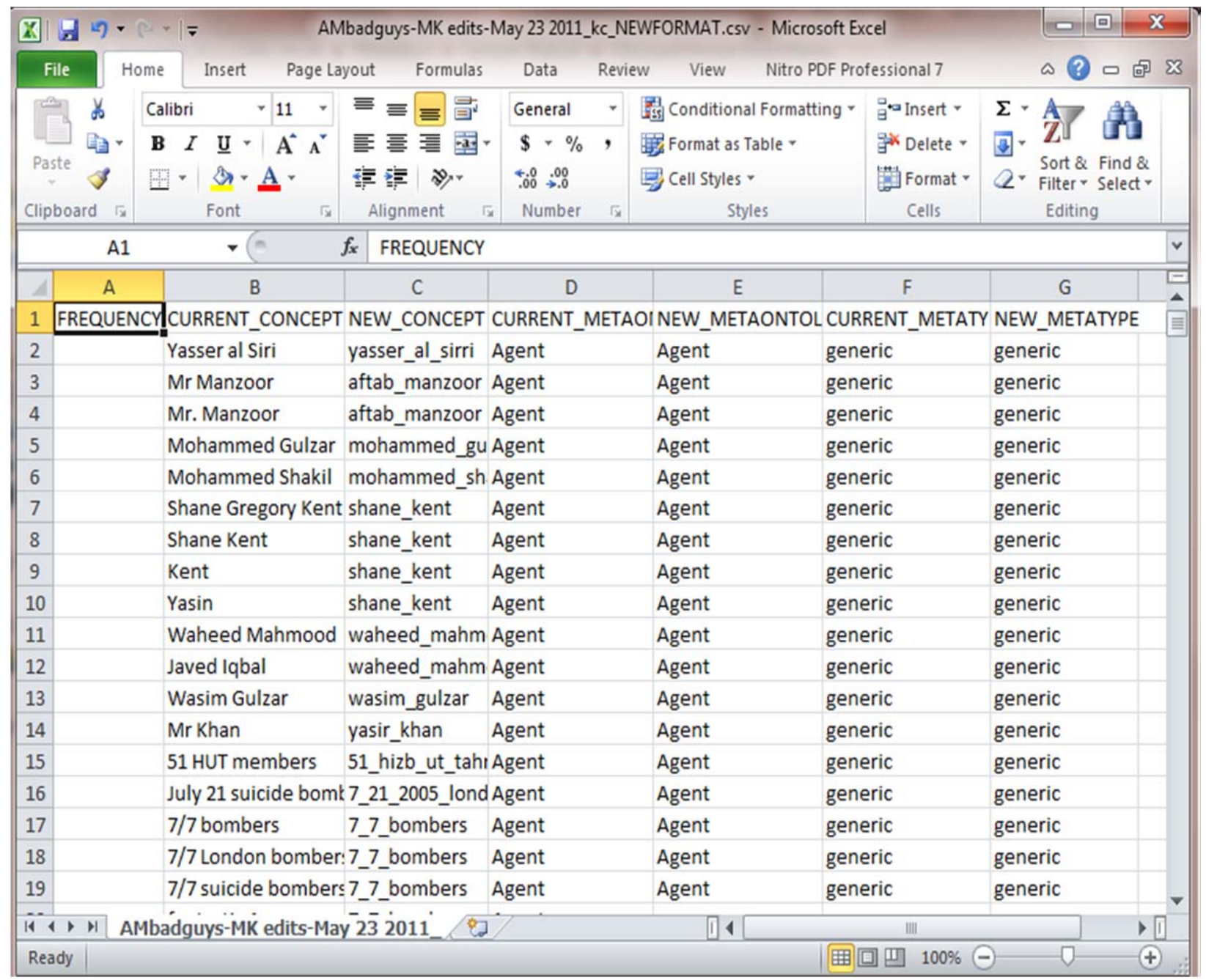

Fig 7: Reduced format thesauri. 


\subsection{Change Format}

Comprises of 10 columns and subsumes all the previous formats of Thesauri. Out of these 10 columns, seven columns are same as that of the reduced format. The three other columns are: casosWhat, casosWhy, and number_of_texts. The casosWhat column contains the name of the thesaurus file from where the concept comes. The casosWhy column essentially is an explanation for the mapping from the Initial concept to the New concept. For example: "Entry created because of depth first search". The number_of_texts column, as the name suggests contains a numeric value relating to how many texts contain the concept.

Note: There can be other columns also, apart from the ones that are listed. For example: TF-IDF, EPA etc.

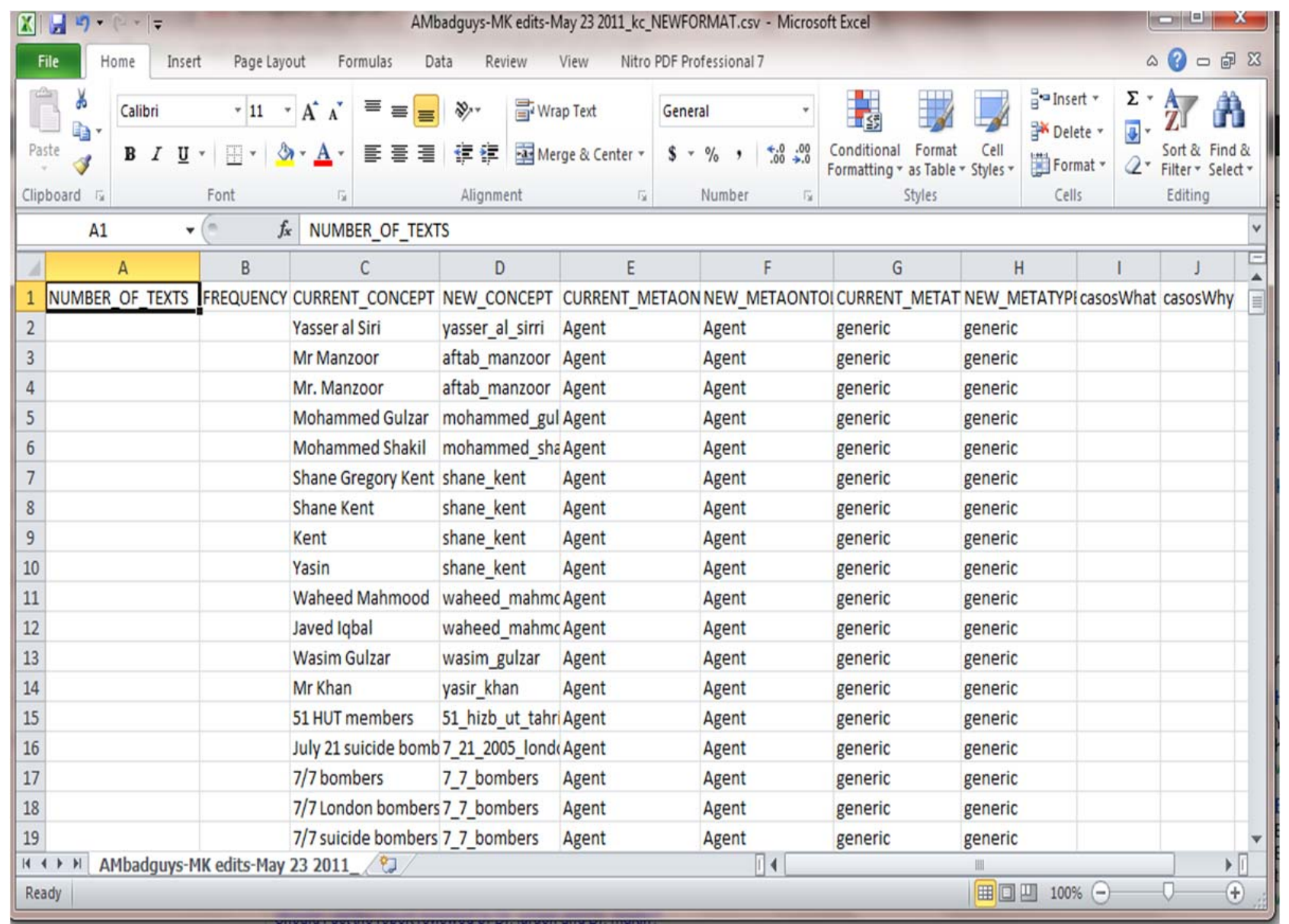

Fig 8: Changed format thesauri. 


\section{Evolution of the AutoMap Thesaurus}

Over a period of years and as part of the process of making network analysis more detailed and informative, the format of the thesaurus has evolved according to the needs of the user.

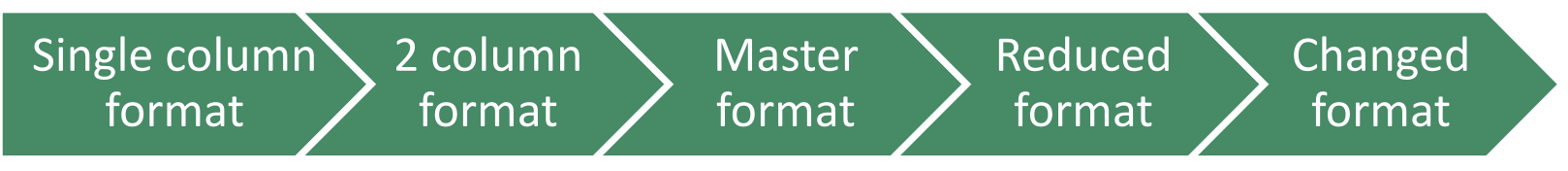

Fig 9: Evolution of Thesaurus

1. Single column format: One of the earliest types of the thesaurus file used in the Automap for network analysis. File using this format remain in use for delete lists in AutoMap. It supports deleting unneeded concepts from the raw data.

2. Two column format: The two column format was an elaboration over the single column type format. This format is usually used for two types of thesauri, namely, 2 column generalization formats and 2 column meta network format. The 2 column generalization format basically relates the concepts from the data set to more abstract concepts. Whereas, the 2 column meta network thesaurus relates the concepts in the data to their ontology.

3. Master format: Master format combines the two types of the 2 column formats and contains 4 columns and adds meta name (or meta type) information. The four columns are conceptFrom, conceptTo, metaOntology, metaName. The master format can be used as a delete list and a meta network thesaurus as well.

4. Reduced format: The reduced format was a more advanced type of format and was basically developed for the purpose of performing editorial and deletion changes easily using ORA [2]. For this purpose, 2 new columns were added to the master format, which were the new metaOntology and the new metaType in addition to the previous ones. Apart from using reduced list as a simple delete list and a meta network thesaurus, it can be used directly in ORA to modify the links and the nodes using the \#delete and \#merge actions. 
5. Change format: At present, the most advanced and subsumes all the previous formats. The changed format is the 10 column format which includes 3 extra columns apart from the reduced format type. These additional columns are casosWhat, casosWhy and

6. NUMBER_OF_TEXTS columns. This format like the reduced format can be used for modifying the links and the nodes directly in ORA [2].

\section{Thesaurus Columns Defined}

In this section, we discuss what each column means in the changed format:

1. NUMBER_OF_TEXTS: This column contains a numerical value which relates to how many times each concept occurs in a corpus of text.For example: If the concept Honda comes in 5 text sets, this column would contain a number

2. FREQUENCY: This column contains a numerical value pointing to how frequent the concept is in a corpus of text data.

3. CURRENT_CONCEPT: The set of words you want converted/ translated into NEW_CONCEPT. The CURRENT_CONCEPT can be a phrase in the article you are coding or a concept in dynetml.

4. NEW_CONCEPT: These are the words/ n-grams which are mapped from the CURRENT_CONCEPT column, these entries are the used as the final concepts in the Meta network. The blanks are converted into underscores in the new concept column. No capitalization is present in this column, and there should be no symbols.

5. CURRENT_METAONTOLOGY: This column is generated by the . AutoMap classifies the concept term into one of the Meta Ontologies values or leaves it blank. The allowable meta-ontology terms are: agent, organization, location, task, event, knowledge, resource, belief.

6. NEW_METAONTOLOGY: If the ontology class deduced by AutoMap is wrong then put the right class value in the column NEW_METAONTOLOGY. If the concept under CURRENT_CONCEPT should be deleted and not coded then under NEW_METAONTOLOGY put \#delete. If the phrase under CURRENT_CONCEPT should not be treated as a phrase but split into its parts then under NEW_METAONTOLOGY \#split. 
7. CURRENT_METATYPE: This column specifies the type for CURRENT_CONCEPT as defined by AutoMap. It is either "generic" or "specific" or blank. Note ONLY agent, organization, location, event can have a type. The various metaOntology listed above can be classified into generic and specific type. For example: John Doe is a specific agent whereas policeman is a generic agent.

8. NEW_METATYPE: If the type deduced by the AutoMap seems wrong or if there is no type and the concept is an agent, organization, location, event then the user can add specify the type under NEW_METATYPE.

\section{Meta Ontologies}

The Ontology is defined as the group of classes in which almost every concept and phrase can be classified depending on the context in which the concept/phrase is used in a particular sentence or a paragraph. For example: USA can be a location or an Organization, depending on the context of the data.

\subsection{Standard Node Classes}

In detail, the ten entity classes can be defined as follows:

1. Agents are individual decision makers. The most common type of decision makers is people. However, this category could also be used for other types of actors such as robots or monkeys. A single Agent represents any person: a family member, a Roman soldier, Soothsayer, Calpurnia, Cicero, any historical figure, a terrorist, or a teacher.

2. Resources are products, materials, or goods that are necessary to perform Tasks, Events, and Actions. A Resource could literally be a dagger, a cloak, a crown, a short sword, a computer, money, bombs, tools, or books.

3. Knowledge describes cognitive capabilities and skills. Knowledge could be Trigonometry, History, English, Economics, the science of DNA, or the knowledge about how to perform a surgery or to build bombs.

4. Tasks are well defined procedures or goals. A Task could be any process in a company, e.g. product development or administration, but also the plan to kill Caesar.

5. Organizations are collectives of people that try to reach a common goal. An Organization could be a specific company, the United Nations, or the government of a country.

6. Locations are geographical positions at any aggregation level that describe places or areas. A Location could be 1600 Pennsylvania Avenue in Washington, London, Florida, The Middle East, Earth, or Mars. 
7. Events are occurrences or phenomena that happen. An Event could be 9-11, the JFK Assignation, the Super Bowl, a wedding, a funeral, or an inauguration. Specific events are one time occurrences with a specific date.

8. Actions are specific activities done by agents. An Action could be buying a car, writing a letter of recommendation, or flying to Africa.

9. Beliefs are any form of religion or other persuasion. A Belief could be to believe that there is a god, or that there are many gods, or that that Earth is flat. Some beliefs are signaled by sentiment such as "war is bad."

10. Roles describe functions of individual decision makers abstracted from specific Agents. A Role could be leader of a group, driver of a car, or mother of an Agent.

11. Groups also referred to as meta-nodes, are any categorization of nodes into a cluster. Groups are defined by one or more of the grouping algorithms.

To repeat the function of these node classes and to show their differences as well as give a first impression about how these node classes are connected with each other, we summarize the Julius Caesar plot very briefly. Brutus (Agent) and other senators (Roles) agree that Rome (Location) would be a better place without Caesar (Belief). To kill Caesar (Task) they form a group of assassins (Organization). To accomplish their task they need to know about Caesar's daily routine (Knowledge) and how to get their knives (Resources) into the senate. Finally, the assassination (Event) takes place because somebody actually stabs Caesar (Action). 


\subsection{Actions}

Apart from the various meta ontologies, the NEW_METAONTOLOGY column in the reduced format and the change format can contain some actions which help in rearranging the links and managing the nodes directly in ORA [2]. These actions help the users to manually edit the thesaurus according to their own needs.

1. Delete: This is actually an action rather than an ontology item, but this is placed in the Meta ontology column for the convenience of the user. The user can delete an entry by writing \#delete in the New Meta ontology column if the user wishes to delete the entry. When the \#delete tag is added to the column, that concept is deleted from the text.

2. Ignore: This is also an action rather than a Meta Ontology value. Ignore is used if the set of concepts should be treated as individual words. The user can use this facility by writing \#ignore in front of the entry which has to be ignored. When the \#ignore tag is added to the metaOntology column, that particular concept is ignored.

3. Split: This is also an action similar in usage to the Delete and Ignore actions. If a group of words are mapped as a phrase, and if the user wants the words in phrase to be independent concepts, then the user can add \#split tag in the New_metaOntology column.

\section{Generic and specific}

The generic and specific are the two allowed metaType values. They provide the users with some additional information on whether this is a universal or general concept or a specific concept. The difference between Specific and Generic can be simply put as the difference between a and the.

1. Specific: Concepts which reference a specific agent, location or organization. For example: John Doe is a specific agent, CASOS is a specific organization, and USA is a specific location.

2. Generic: concepts which are universal and do not point to a specific agent, location or an organization. For example: man is a generic agent, health fund organization is a generic organization, and my room is a generic location. 


\section{Delete Lists}

Delete lists allow the users to remove non-content bearing conjunctions, articles and other noise from texts. Delete lists can be created internally in AutoMap or externally in a text editor. The list itself is a text file that contains a list (one concept per line) of the words to be deleted from the text.

Note: Whether the users apply the Delete list(s) before or after applying a Thesaurus will depend on their individual needs.

\subsection{Domain delete list}

A domain delete list is one which contains concepts which need to be deleted and are project specific.

Note: \#delete in the change format moves the CURRENT_CONCEPT to the domain delete list.

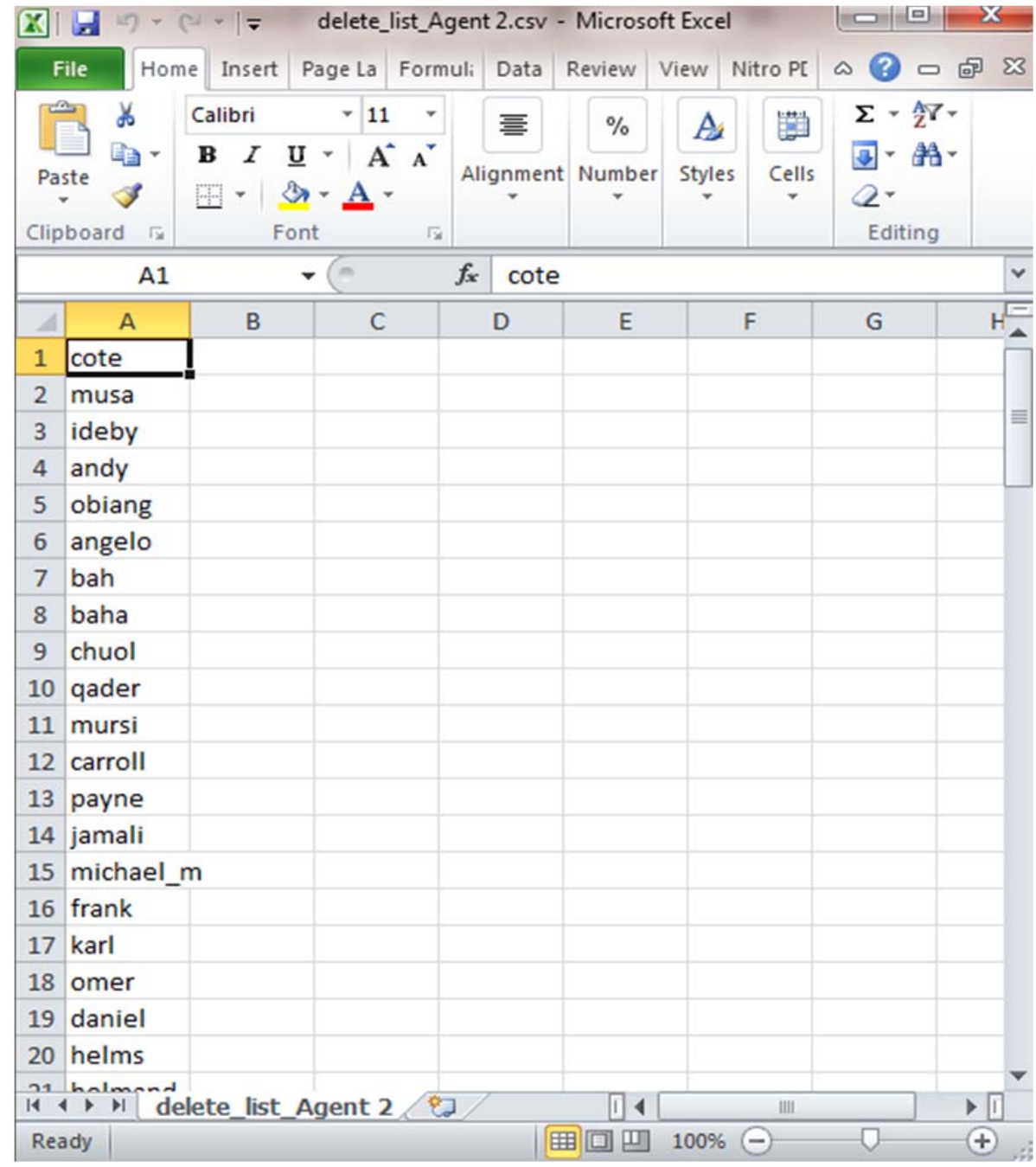

Fig 10: Domain delete list 


\subsection{Universal delete list}

A universal delete list contains concepts that need to be deleted and are generally comprised of the universal concepts like pronouns, months, numbers and other noise words.

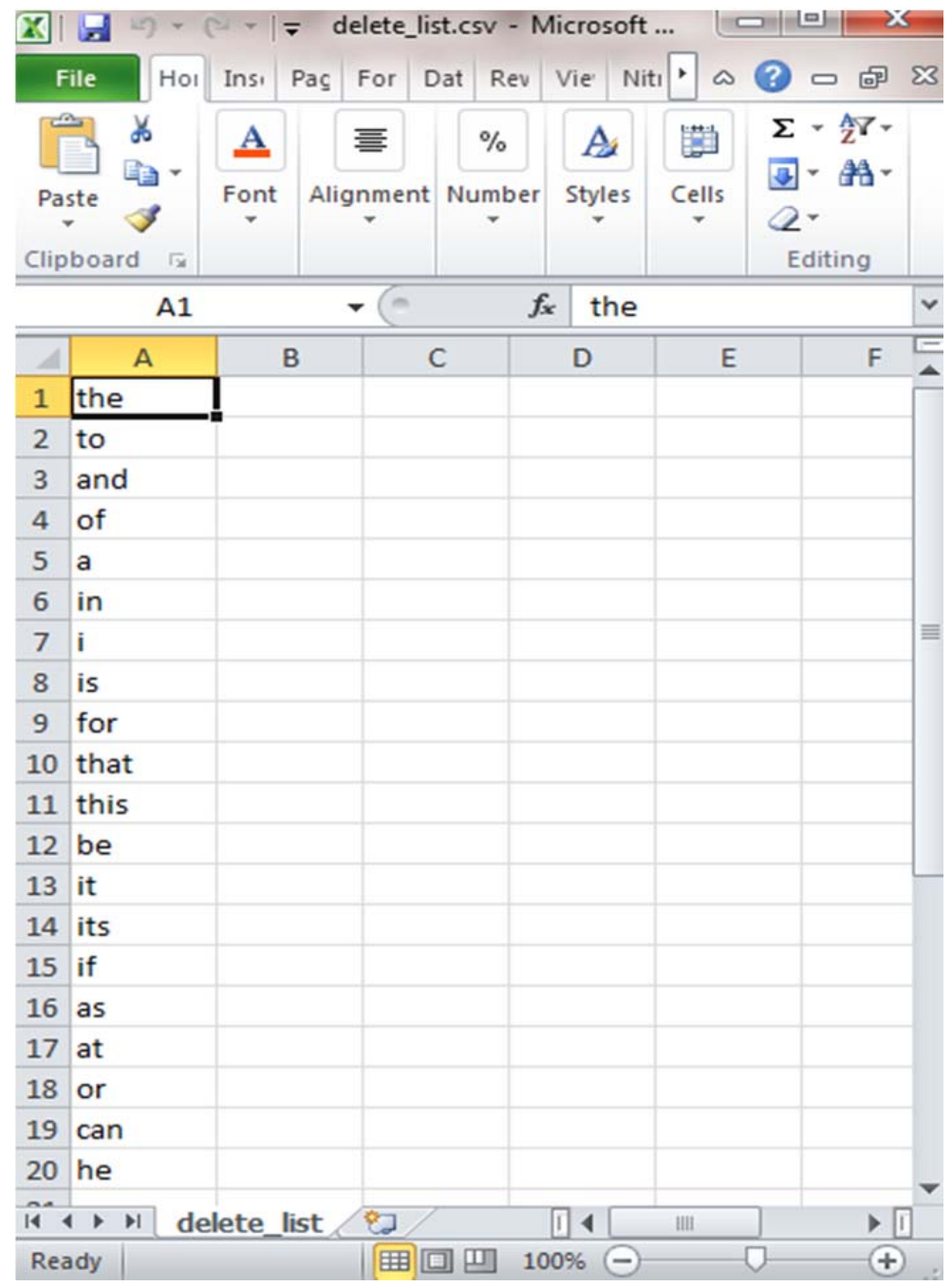

Fig 11: Universal delete list 


\section{Difference between the Domain Thesauri and the Universal Thesaurus}

The product of the thesaurus creation process (i.e. the outcome from splitting and merging) can be classified into two major types of thesaurus files. The Universal/Master thesauri is intended for general application to all input text sets and the domain thesauri which contains concepts which are project specific and hence is used on specific text sets and not on others.

\subsection{Universal Thesaurus}

This is the major thesaurus which is created by the merge and split process. This thesaurus file contains all the concepts which are not specific to the projects (which are contained in domain thesaurus), the universal thesaurus contains all the common words, acronyms, common nouns, verbs, proverbs etc. Apart from these, the universal thesaurus also contains all the geographical locations and the organizations.

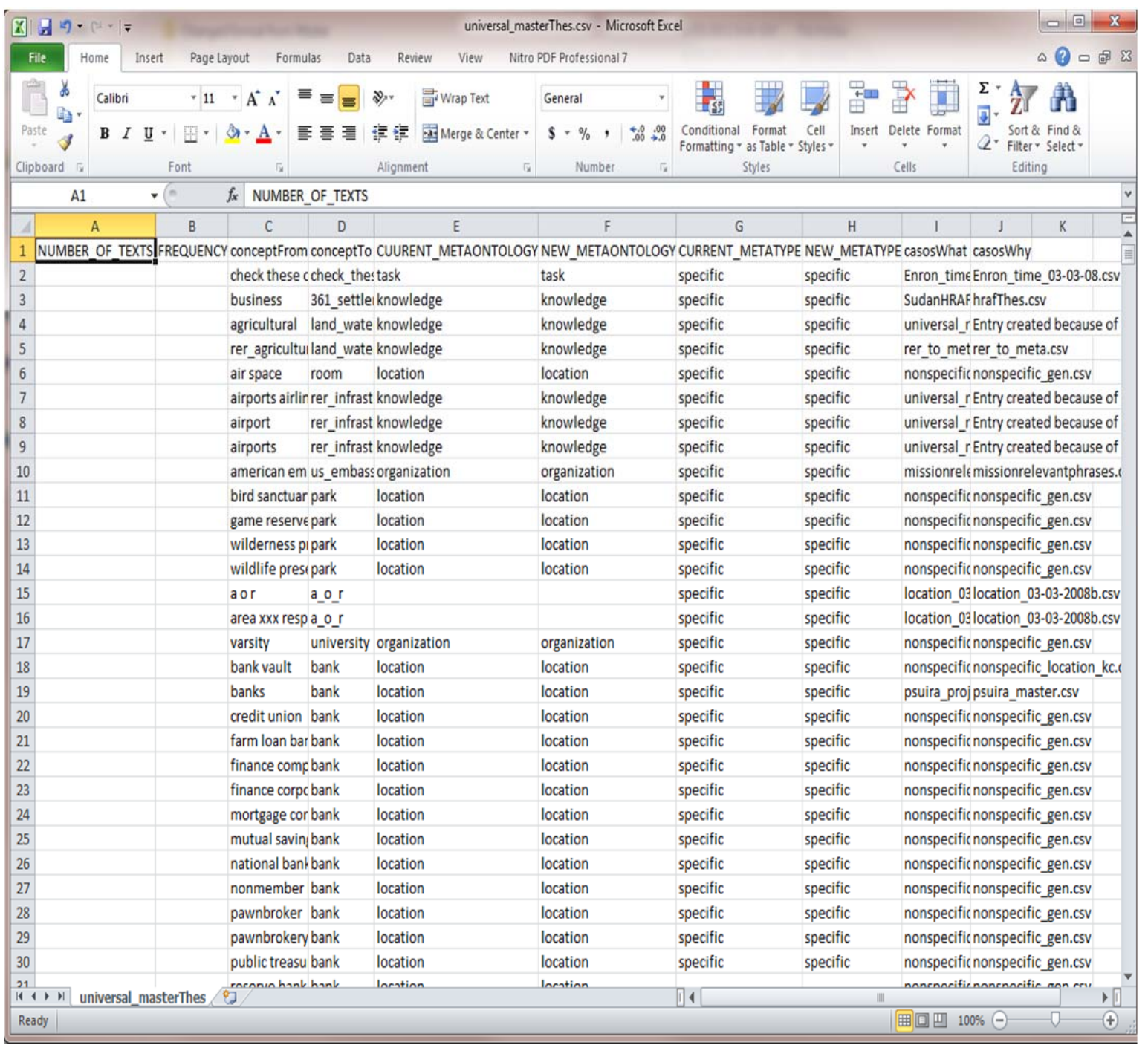

Fig 12: Universal master thesaurus (universal_masterThes.csv) 


\subsection{Domain Thesauri}

The domain thesauri are project specific thesauri produced by the merge and split process. According to our approach, the domain thesaurus contains only agents which are specific to the project. The approach used to differentiate between the project specific agents and the universal agents is that the project specific agents were considered to be the ones which were one word names, i.e. no white spaces were present.

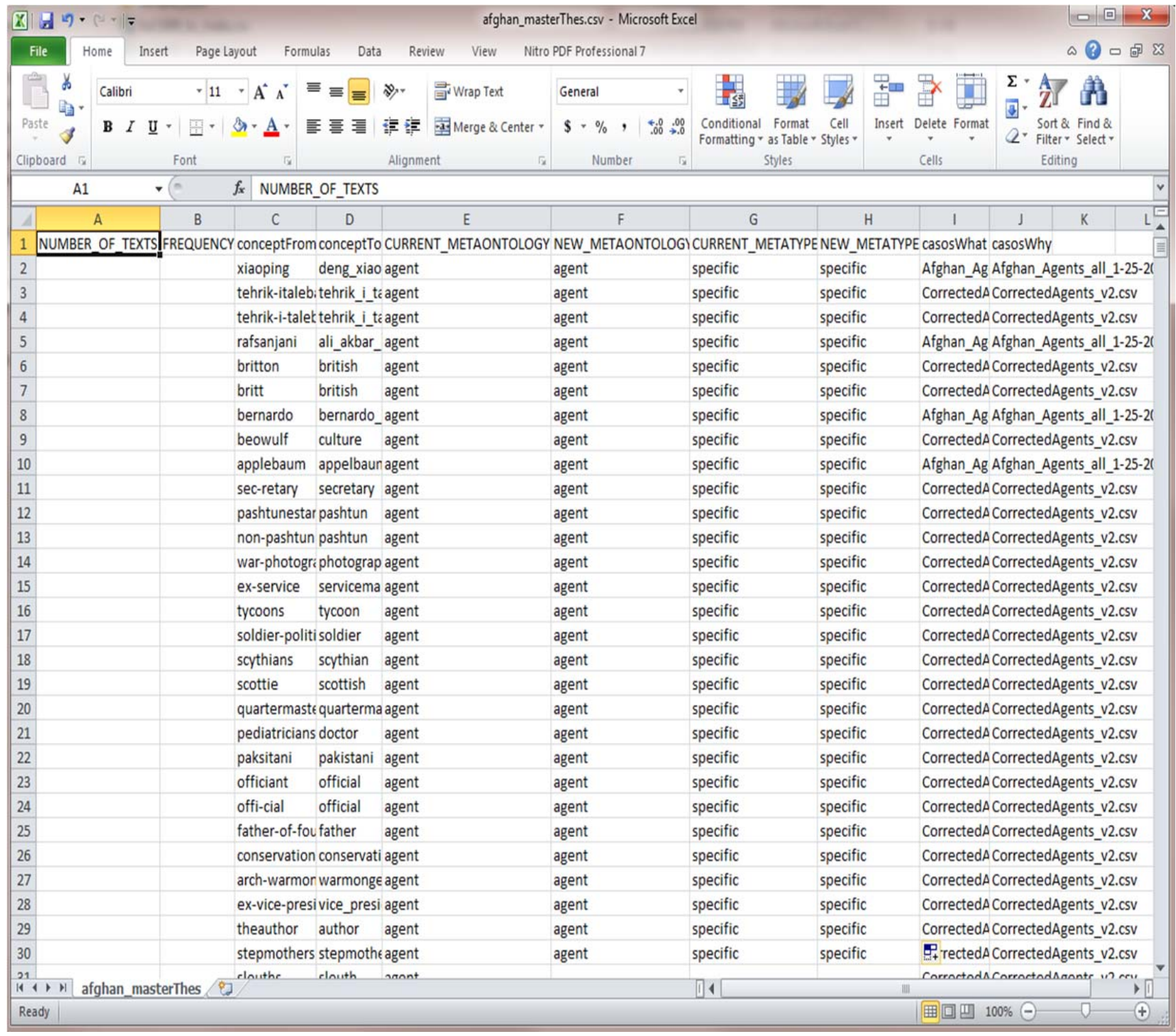

Fig 13: Domain thesaurus (afghan_masterThes.csv) 


\section{Dominance of Domain Thesauri}

Apart from the difference in the content and the data contained by the two thesaurus files, these two types of thesauri differ in the precedence as well. The mapping in the domain thesauri takes precedence over the mapping in the universal thesaurus when both types of thesaurus files are used when performing data analysis by generating a Meta-network out of a corpus of raw text data.

Example: Consider a data set which needs to be analyzed by generating the Dynetml (network) files using AutoMap. In order to generate this network we provide the two thesaurus files (the domain thesaurus and the universal thesaurus). Now, assume that the word Obama appears in the body of text for analysis. AutoMap references the thesauri files to map the concept, and the concept Obama is mapped to barack_obama in the Domain thesaurus and in the universal thesauri, it is mapped as president_of_USA. AutoMap applies mapping which is in the domain thesaurus giving preference to the domain thesaurus over the universal thesaurus. Thus, we say that the Domain thesaurus has precedence over the Universal thesaurus.

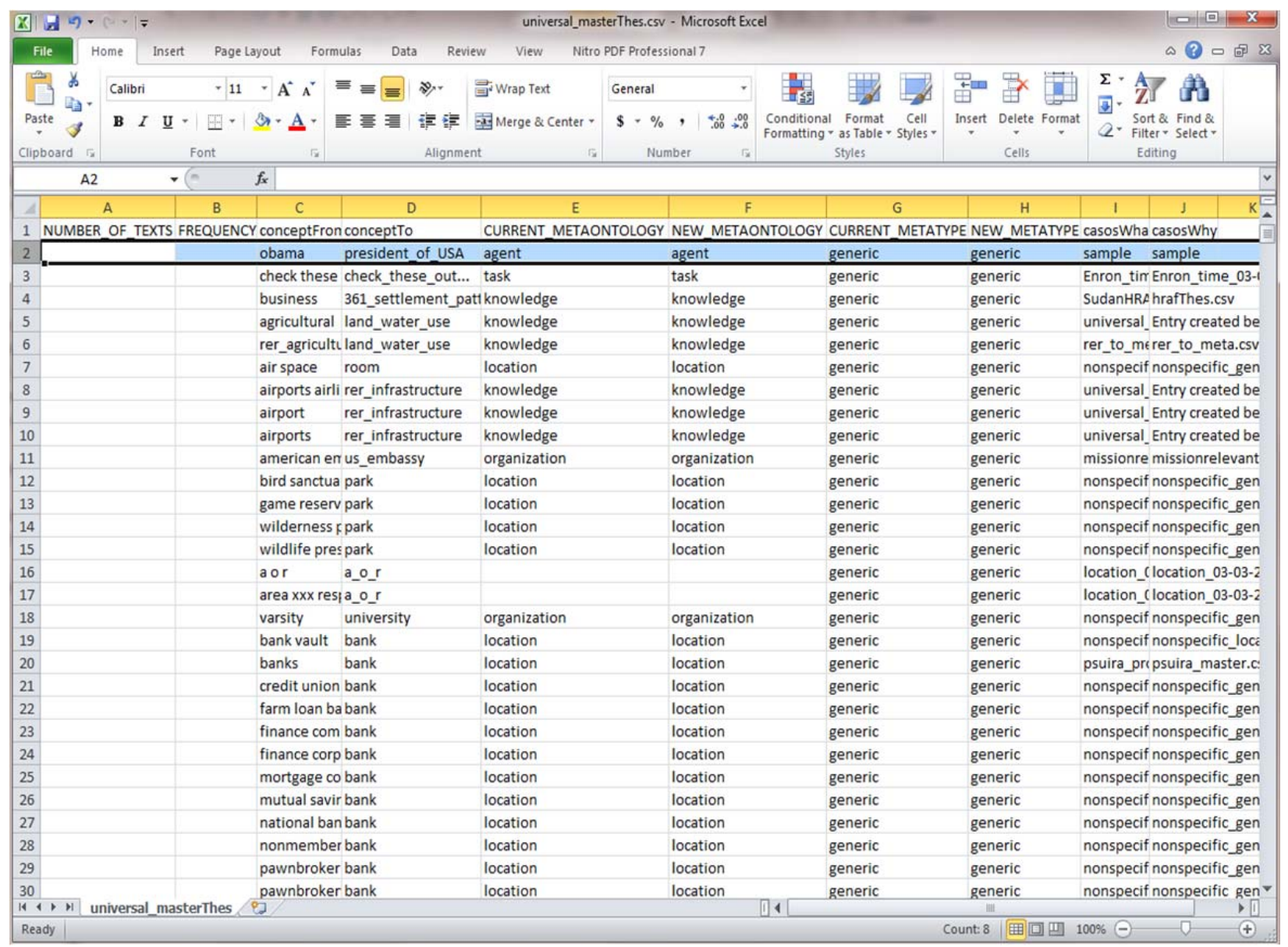

Fig 14: Universal master thesaurus (universal_masterThes.csv) 


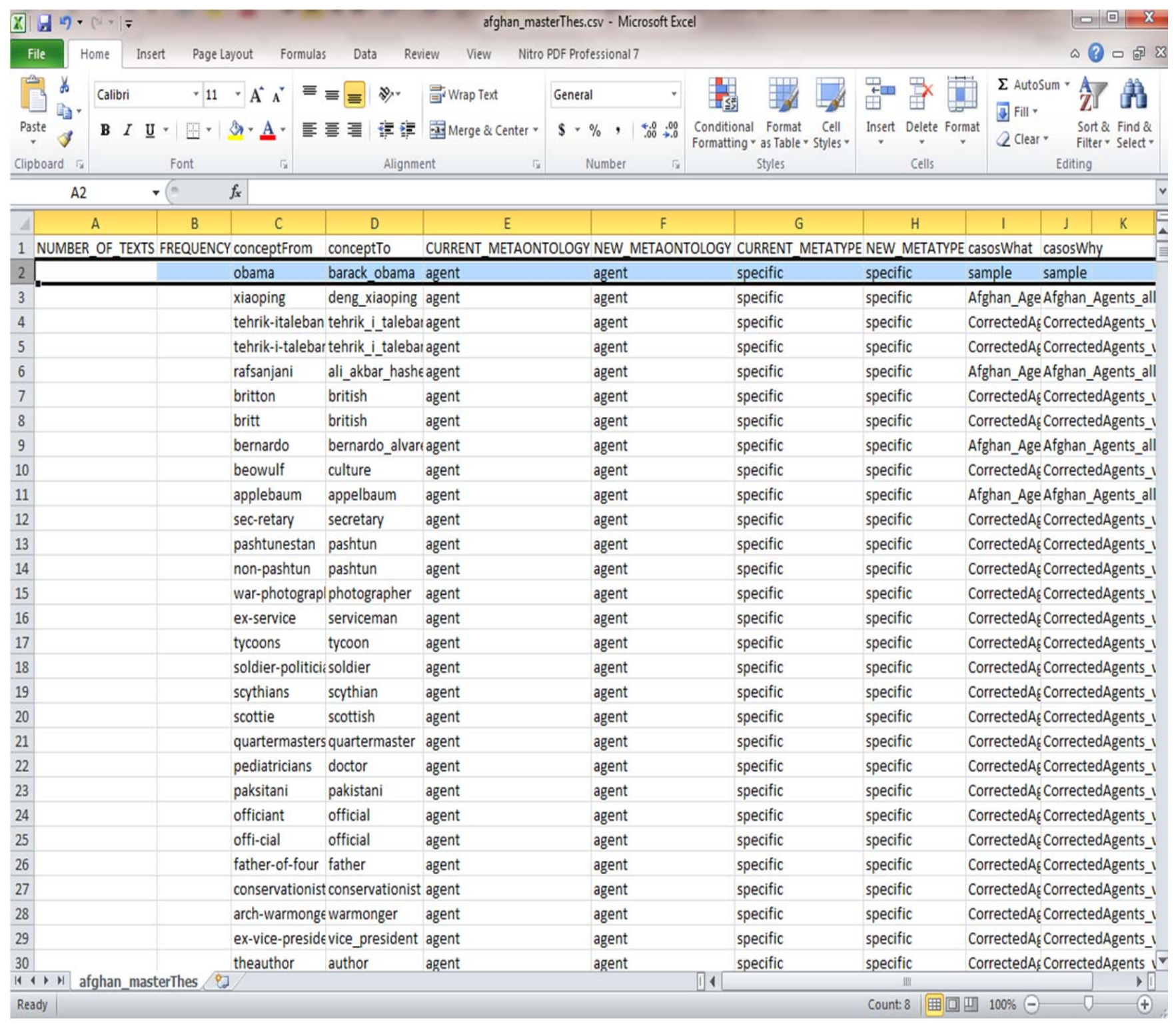

Fig 15: Domain thesaurus (afghan_masterThes.csv) 


\section{The Split Process}

The development copy of AutoMap contains the required files and utilities for thesaurus split and merge. Typically a user will check out a copy of AutoMap from the Subversion repository prior to applying the process. At present this process is not performed using the general distribution copy of AutoMap

The split process used during thesaurus preparation uses these steps:

1. Open the splitThes.bat in notepad in order to explore the file list for the Split process.

2. Run the split process using SplitThes.bat which is located in Automap3lusrletc directory.

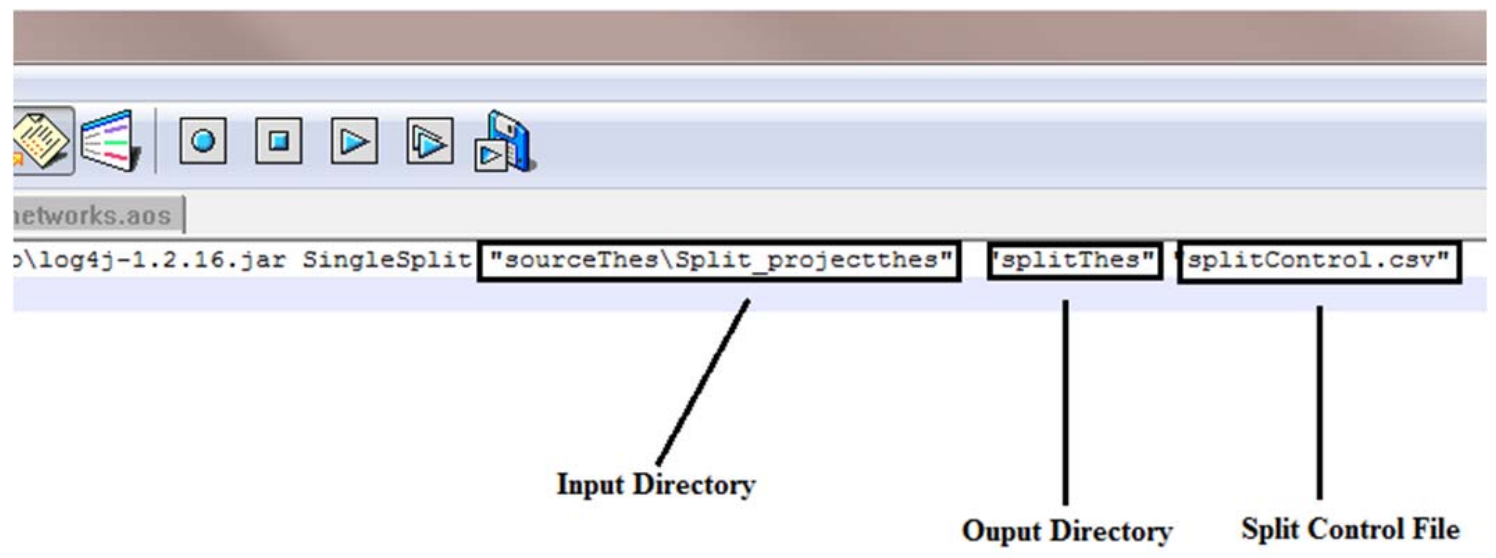

length: 201 lines: 3

$\operatorname{Ln}: 2$ Col: 6 Sel:0

DosıWindows

ANSI

Fig 16: Screen shot of the file splitThes.bat

3. The input directory should contain all the files which need to be split and should be listed in the file splitcontrol.csv

4. The output directory splitThes will contain all the files after the split process is completed.

5. The split control file generally has to be modified by the users to process the specific thesauri needed by the user. The column A contains the list of all the files which need to be split. The column B contains the Meta Ontology value on which the file needs to be split ("agent" has been used in this example). Column $\mathrm{C}$ contains the name of the file where the split entries should be written and the column D contains the name of the file where all other entries need to be written. 


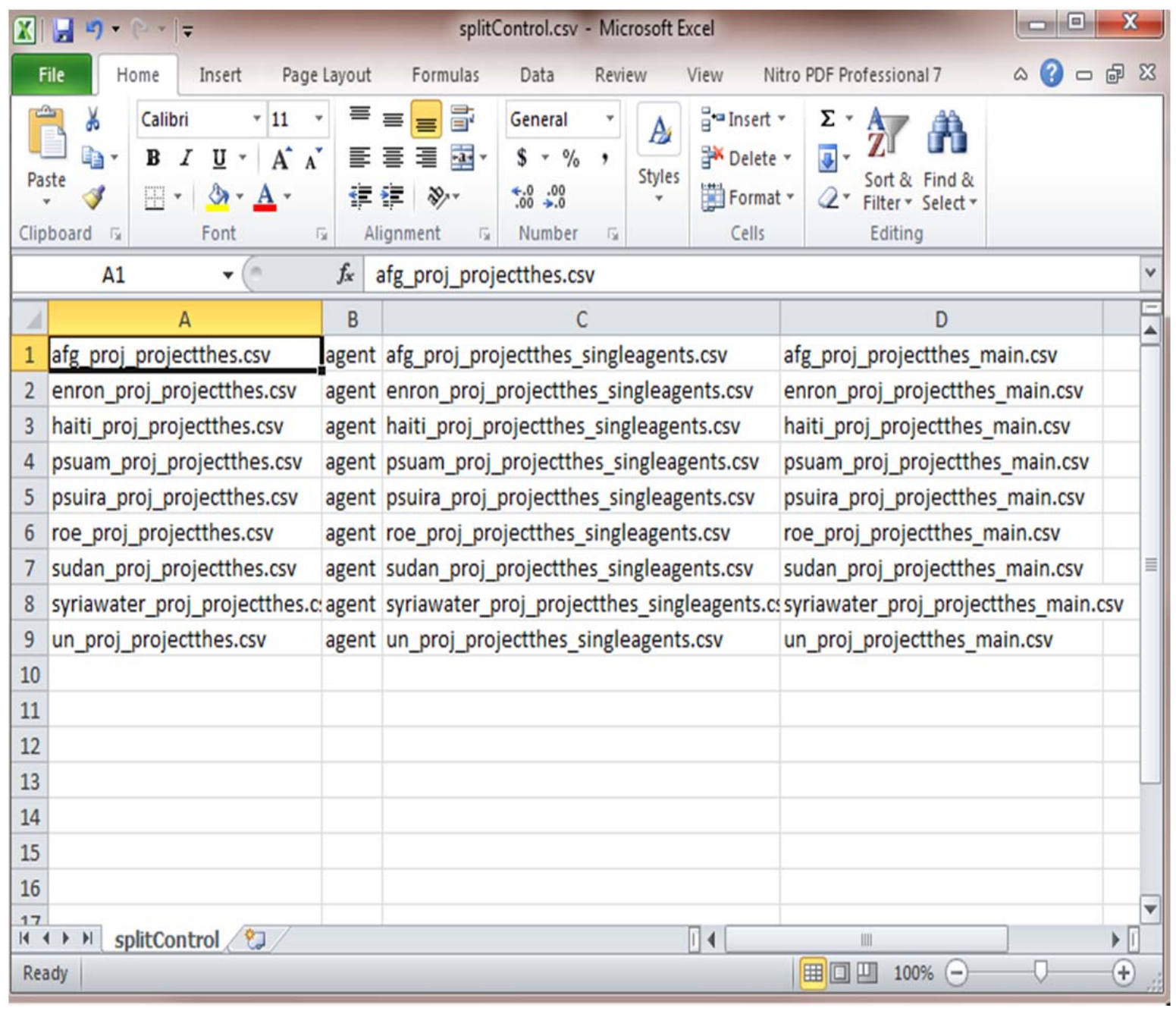

Fig 17: Screen shot for the file splitcontrol.csv

Note: The format used shown above should be strictly used as the process may break down if there are any modifications. The files which need to be merged should be in the master thesauri format. 


\section{Split Routine}

The split routine helps in the splitting of the entries in a file on the basis of an algorithm. The split process used in this report splits the files if the meta ontology of the entry in a file is an AGENT and the word is a single word (without any white spaces) as discussed in the previous section.

Steps involved in the code of the split routine:

1. Opens the file splitcontrol.csv and processes the input line by line.

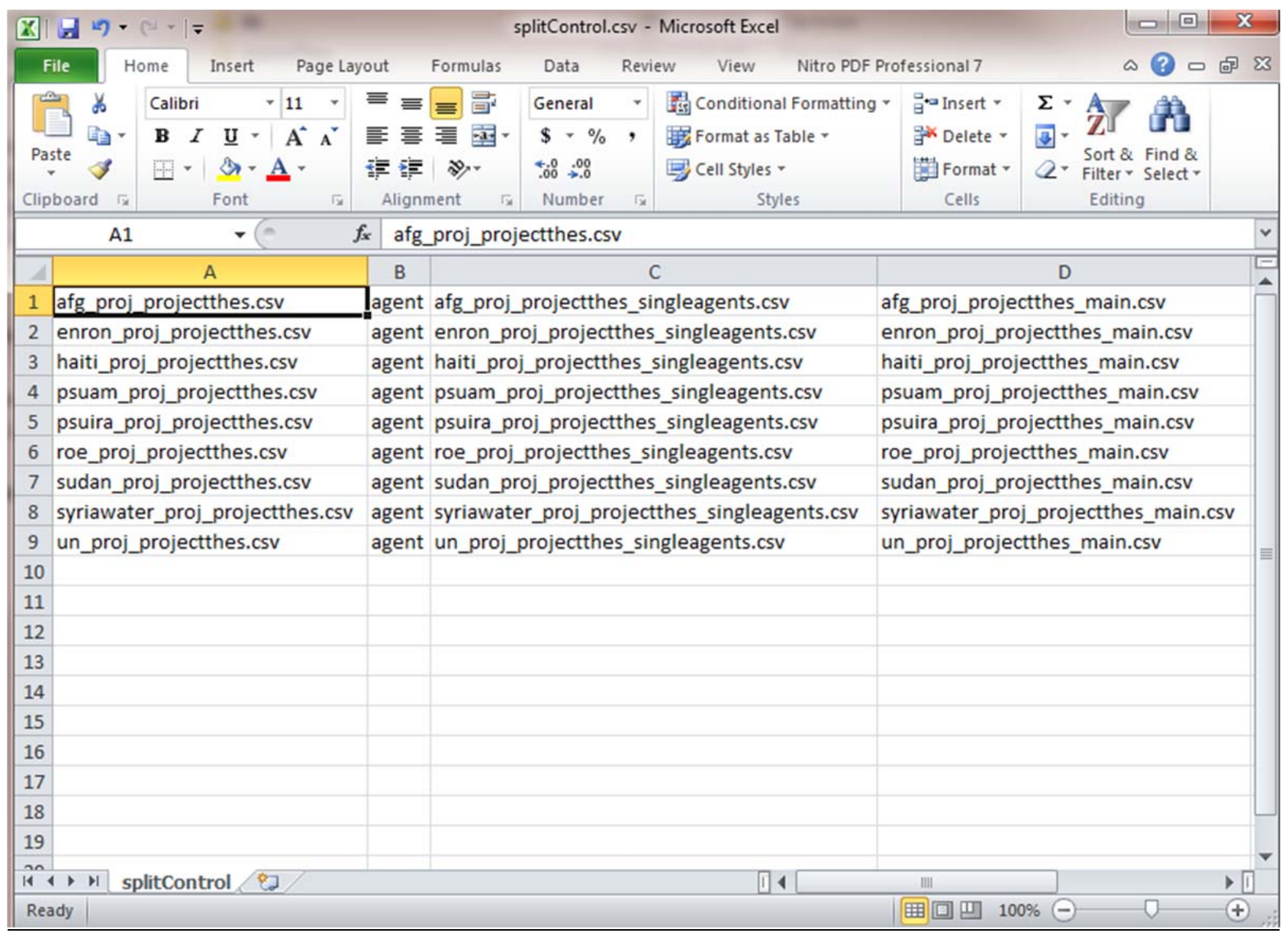

Fig 18: Format for the file splitcontrol.csv

2. Reads the entry in column A and opens the named file. 


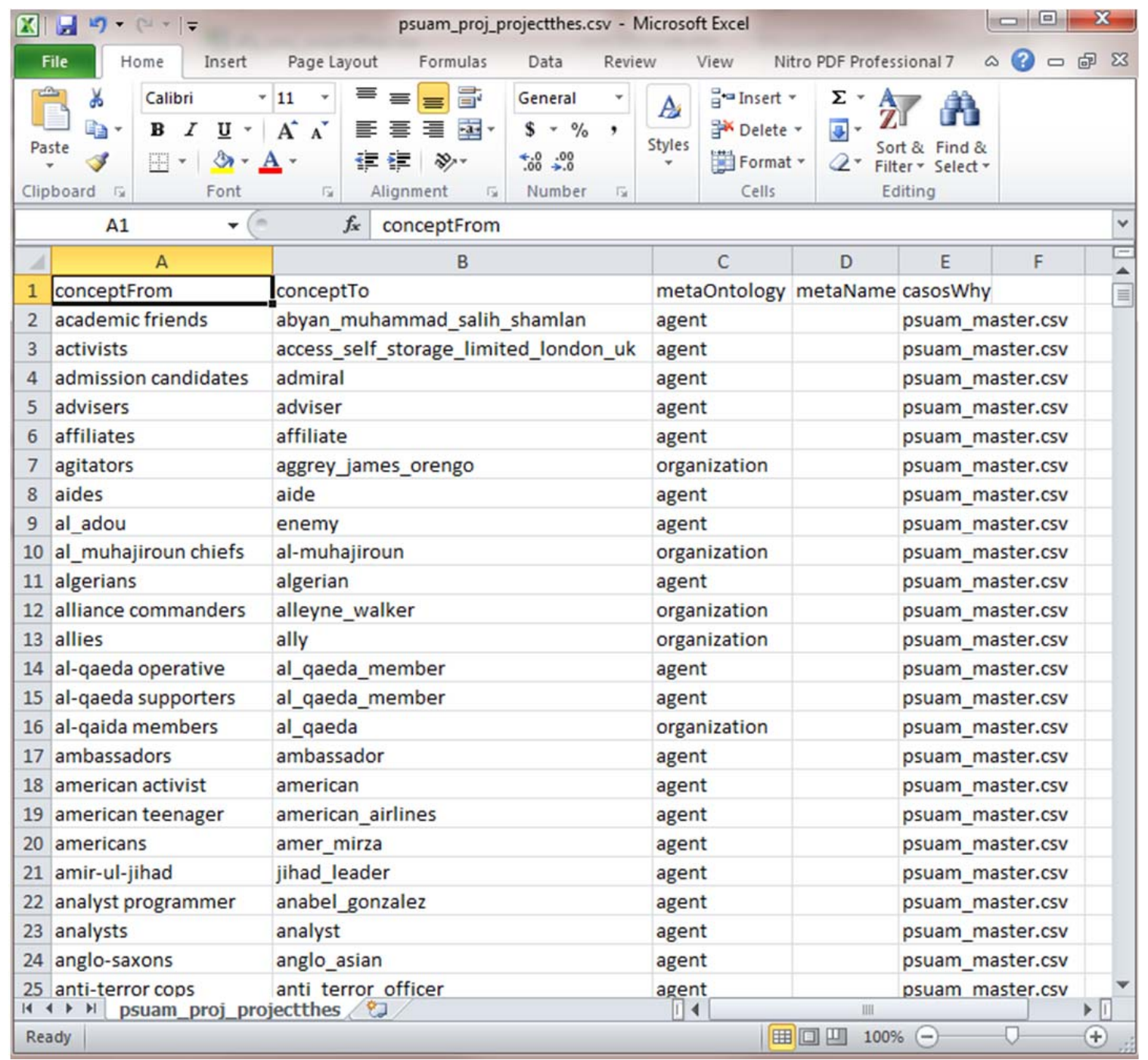

Fig 19: Format for the file in the column A

3. Once the file is opened, the contents are scanned line by line (note however that the header line in the input thesaurus is skipped).

4. The first entry "academic friends" has a white space, so this entry is written to the file name in column D of the psuam_proj_projectthes.csv which is the file psuam_proj_projectthes_main.csv.

5. The second entry "activists" does not have any white space and the corresponding Meta ontology is also agents, so this entry goes to the file named in column $\mathrm{C}$ of the splitcontol.csv, i.e. psuam_proj_projectthes_singleagents.csv.

Note: The split routine can be modified according to the needs of the user by changing the column B of splitcontrol.csv to some other Meta ontology. 


\section{Algorithm for the Split Routine}

This section describes the algorithm for the split process. The algorithm has been simplified as much as possible for the convenience of the reader.

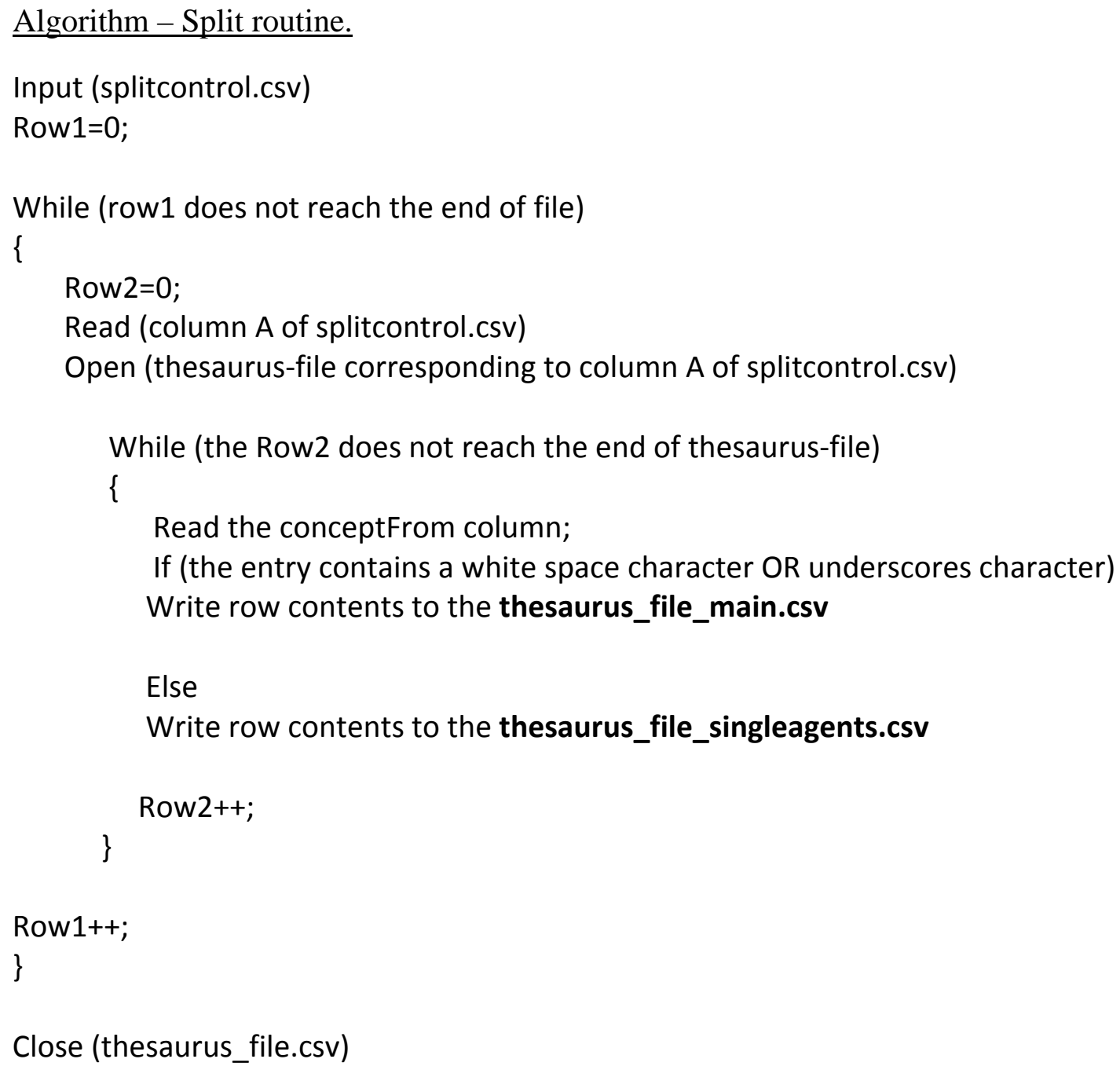




\section{The Merge Process}

The merge routine is not a simple additive process of summing all the concepts from the input files into one thesaurus; it involves some computing operations as well, after we have created a merged thesaurus. These operations are Remove conflicts, Detect cycles, and Depth first search. The merge process used uses the following series of steps:

1. Remove conflicts: After all the input files are merged into one thesaurus file, the function checks for duplicate entries in the conceptFrom column. If an entry is found more than once, it deletes all other occurrences except the first one.

2. Detect Cycles: This function checks if any cycle is created due to merging all the input files. If a cycle of concepts is created, it just maps the concept to itself. For example: Consider that India is mapped to country, country to world, world to tradition, tradition to India. This means that consequently India is mapped to itself, so this function simply matches India to India.

3. Depth first search: This function essentially checks all the chain mapping involved in the merged file. Once the end point of chain mapping is found, it simply creates a mapping from the head to the tail. For example: AutoMap is mapped to Network Analysis, Network Analysis mapped to CASOS, CASOS mapped to CMU. So, this function simply maps AutoMap to CMU.

Note: The order in which these functions appear in the merge algorithm is also significant. 
The steps involved in the Merge process are:

1. Open the mergeStandard.bat in notepad in order to see the list of files and directories employed in the Merge process. (MergeStandard.bat is located in AutoMap3lusrletc directory.)

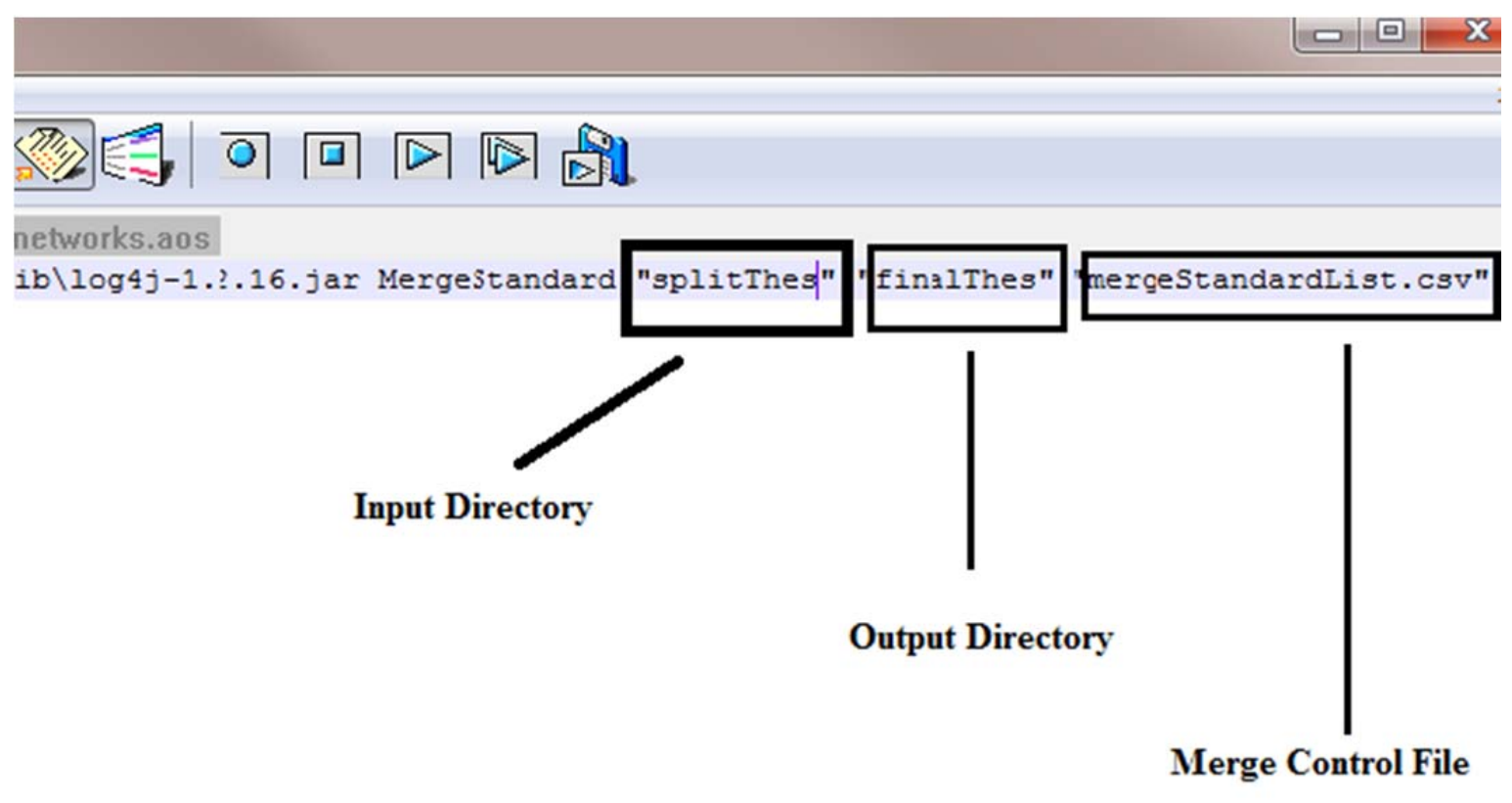

Fig 20: Screen shot for the file mergeStandard.bat

2. The input directory "splitThes" should contain all the files which need to be split. No sub directories are allowed in this directory.

3. The merge control file, mergeStandard.csv controls the merge process. In this file, all the files which need to be merged are listed as well as the category in which they need to be merged is listed. For example: Universal, Sudan, Afghan etc. 


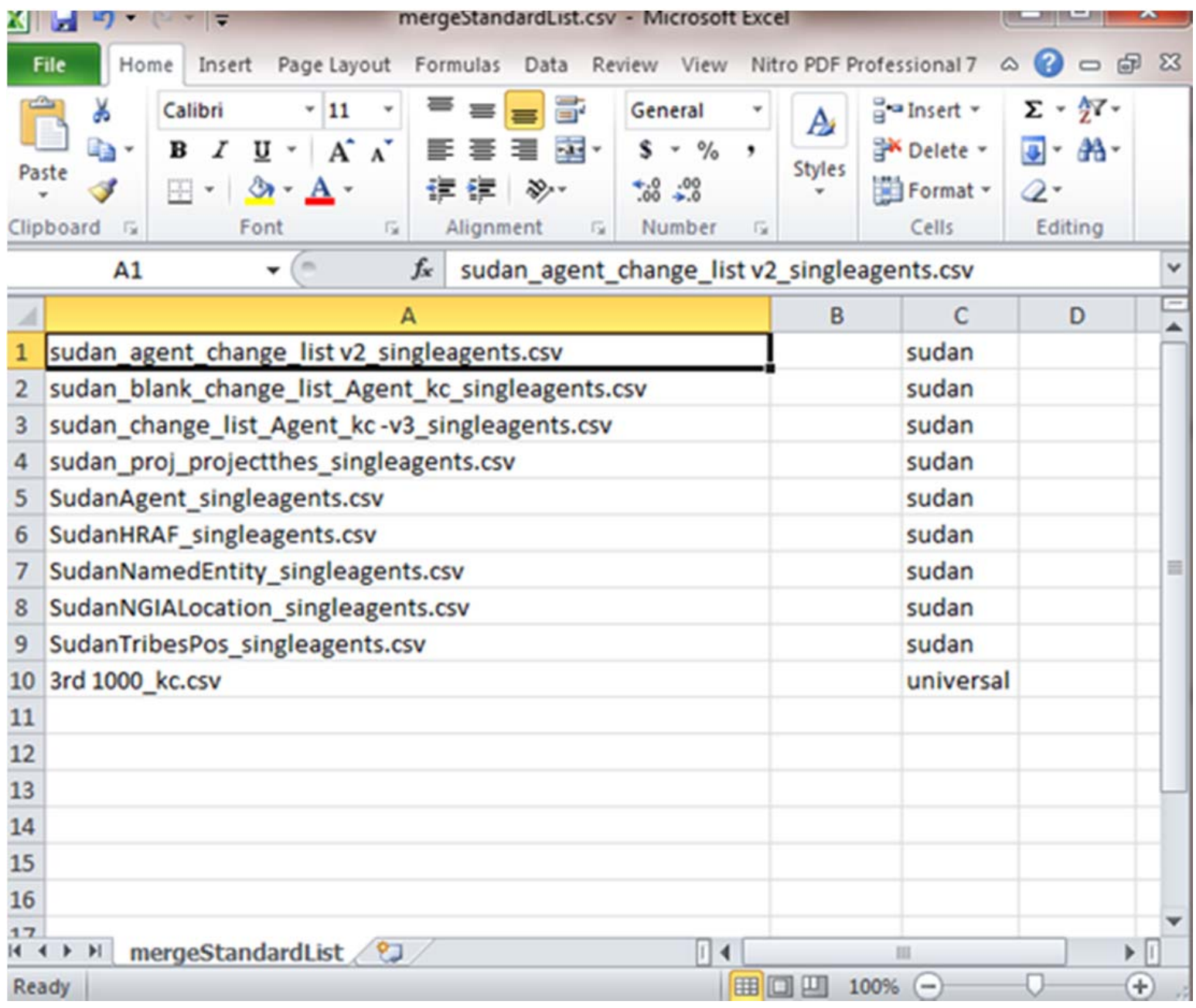

Fig 21: Screen shot for the file mergeStandardList.csv

Note: The Column B of the mergeStandardList.csv can be left as it is not used by merge routine. 


\section{Merge Routine}

The merge routine is used to the merge a group of thesaurus files in a single merged thesaurus file on the basis of an algorithm.

Steps involved in the code of the merge routine are as follows:

1. Opens the file mergeStandardList.csv and scans the input line by line.

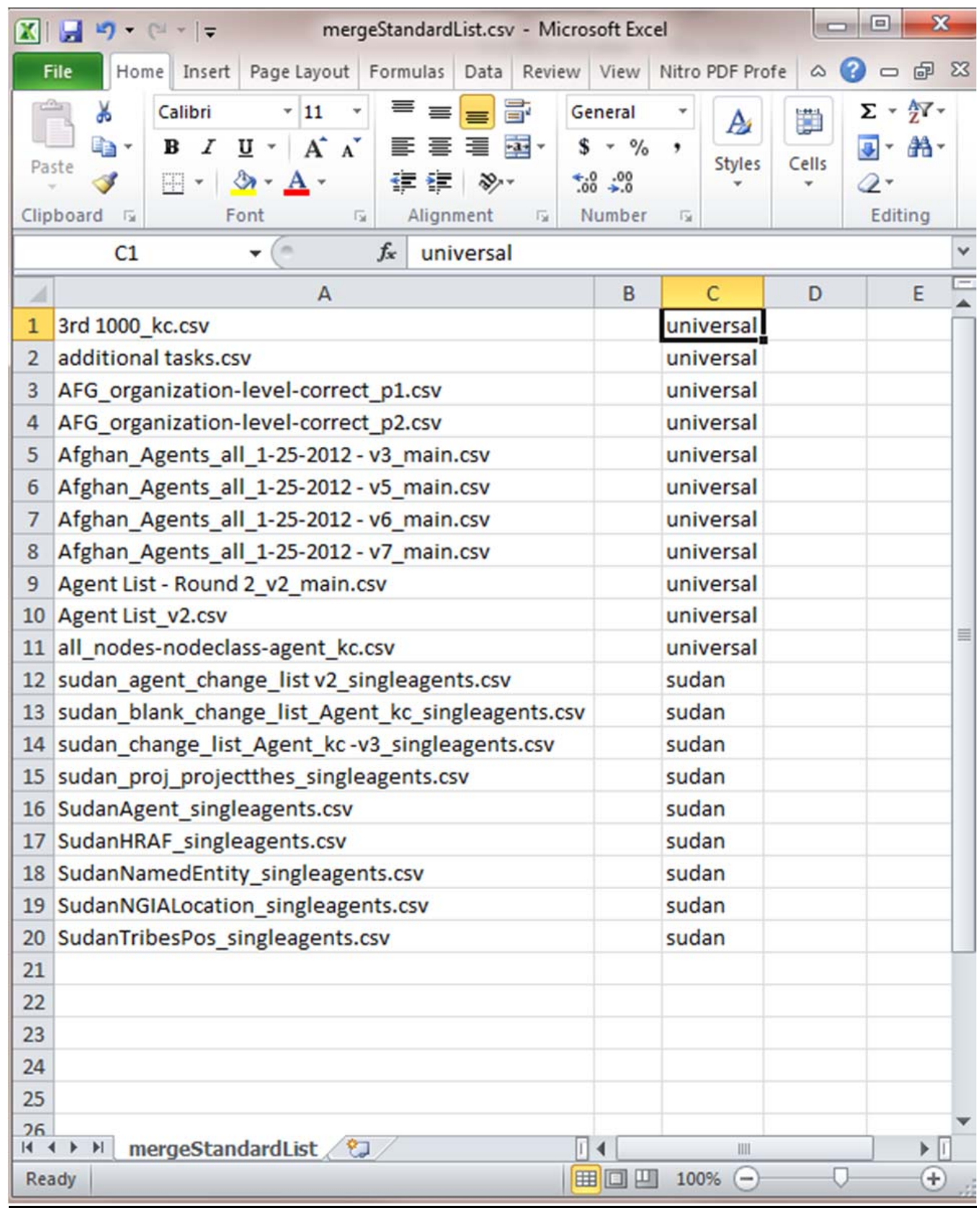

Fig 22: Format for the file mergestandardlist.csv 
2. Reads the entry corresponding to column $A$ and opens the named thesaurus file.

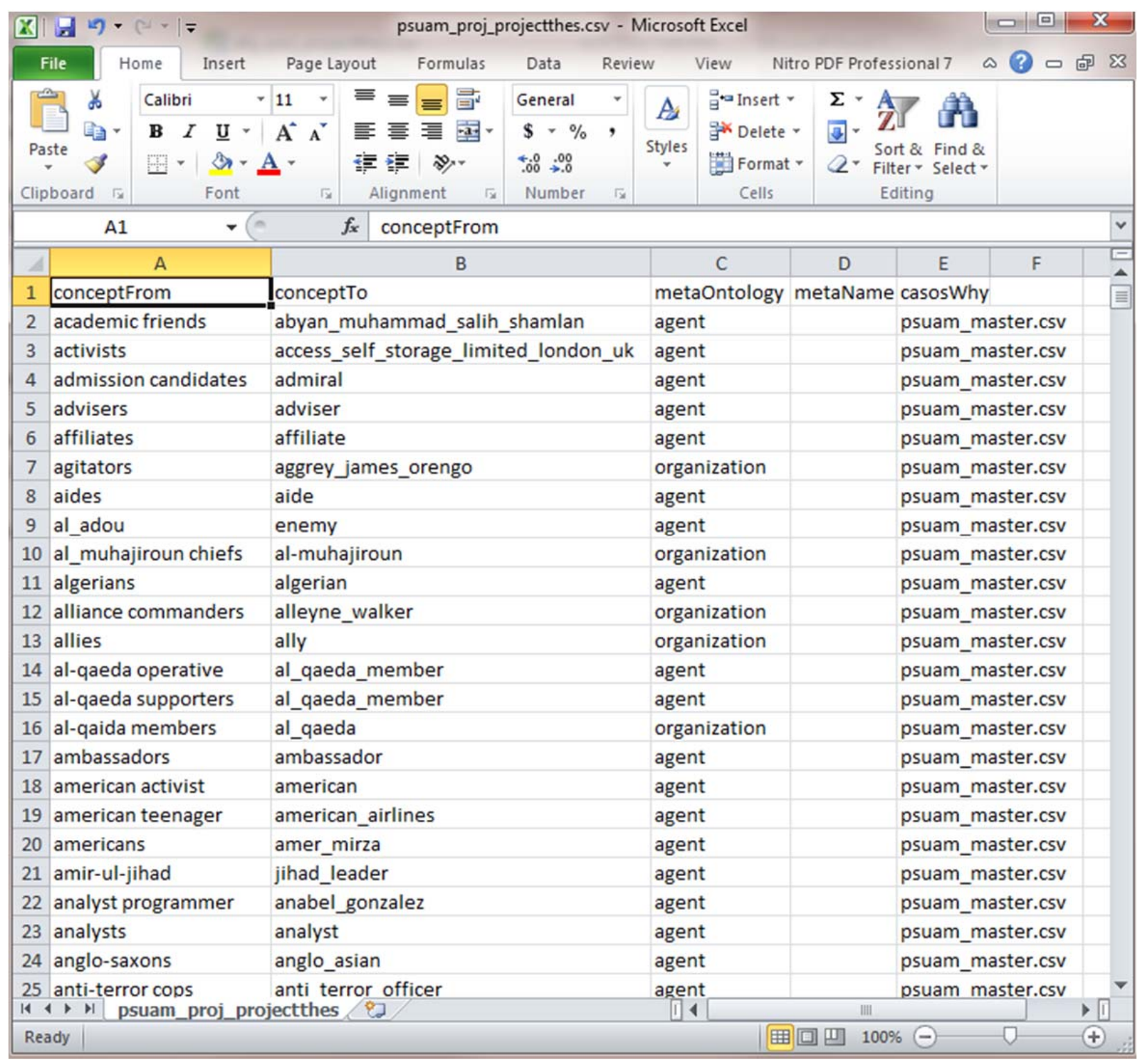

Fig 23: Format for the file in the column A

3. Column $\mathrm{C}$ of the entry line contains "universal”, so all the entries in 3rd 1000_kc.csv are merged into the file universal_masterThes.csv thesaurus file (the output name is $<$ Column C contents > + “_masterThes.csv”).

4. In the 12th line the file sudan_agent_change_list_Agent_kc_singleagents.csv has 'Sudan' in Column C, so all the entries are copied to the sudan_masterThes.csv 


\section{Algorithm for the Merge Routine}

Open (mergestandardlist.csv)

Row1=0;

While (row1 does not reach the end of file)

\{

Row2=0;

Grouping $=$ Read (Column $\mathrm{C}$ of the file mergestandardlist.csv)

Read (Column A of mergestandardlist.csv)

Open (file corresponding to Column A of splitcontrol.csv)

Open (grouping+"'masterThes.csv")

While (the Row2 does not reach the end of file)

\{

Read (the column of concept from);

Write (the entries corresponding to the row)

Row2++;

\}

Remove Conflicts ();

Detect Cycles ();

Depth First ();

\section{Row1++;}

\}

Close (universal_masterthes.csv)

Close (mergestandardlist.csv) 


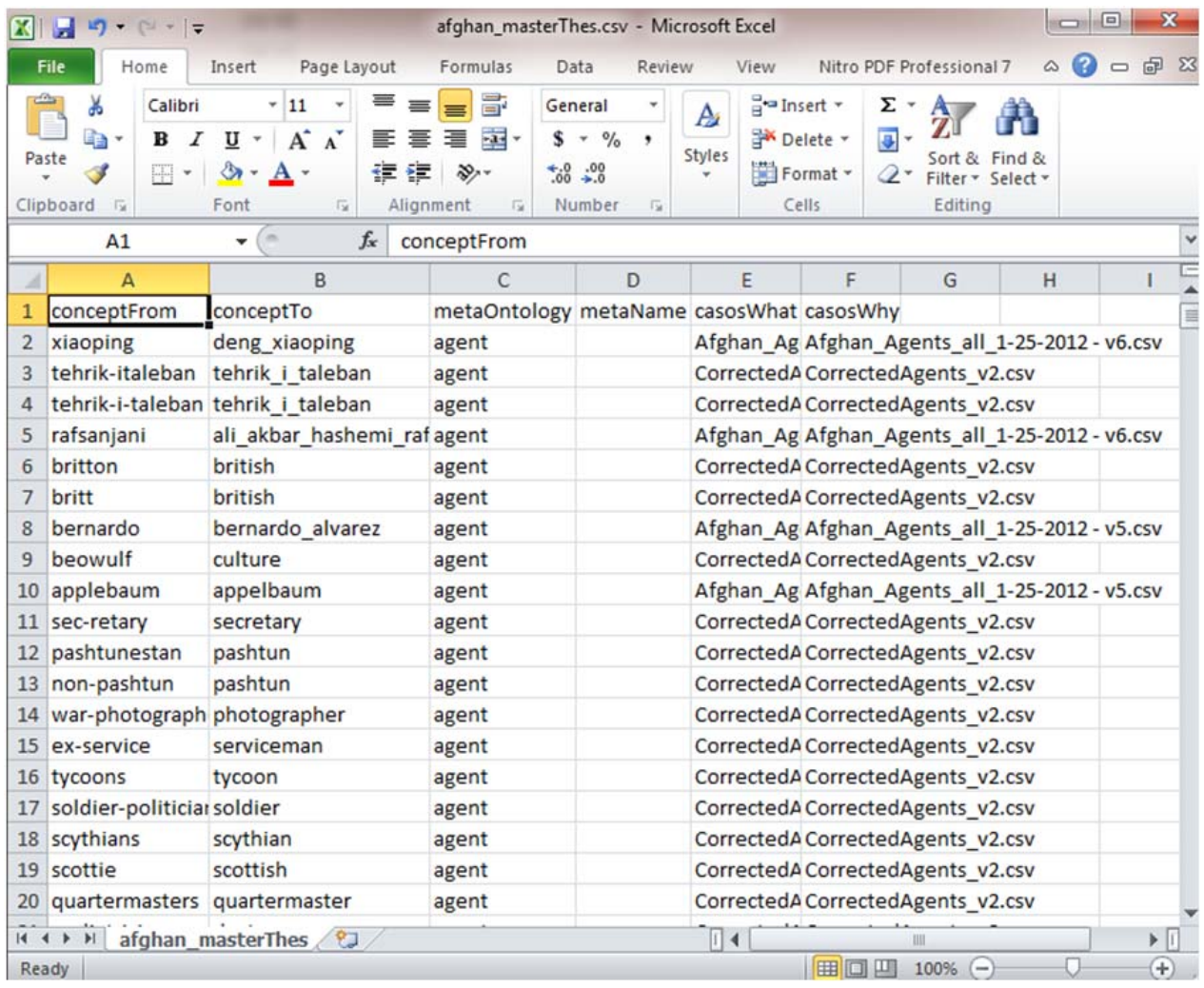

Fig 24: The domain thesaurus after applying the merge algorithm

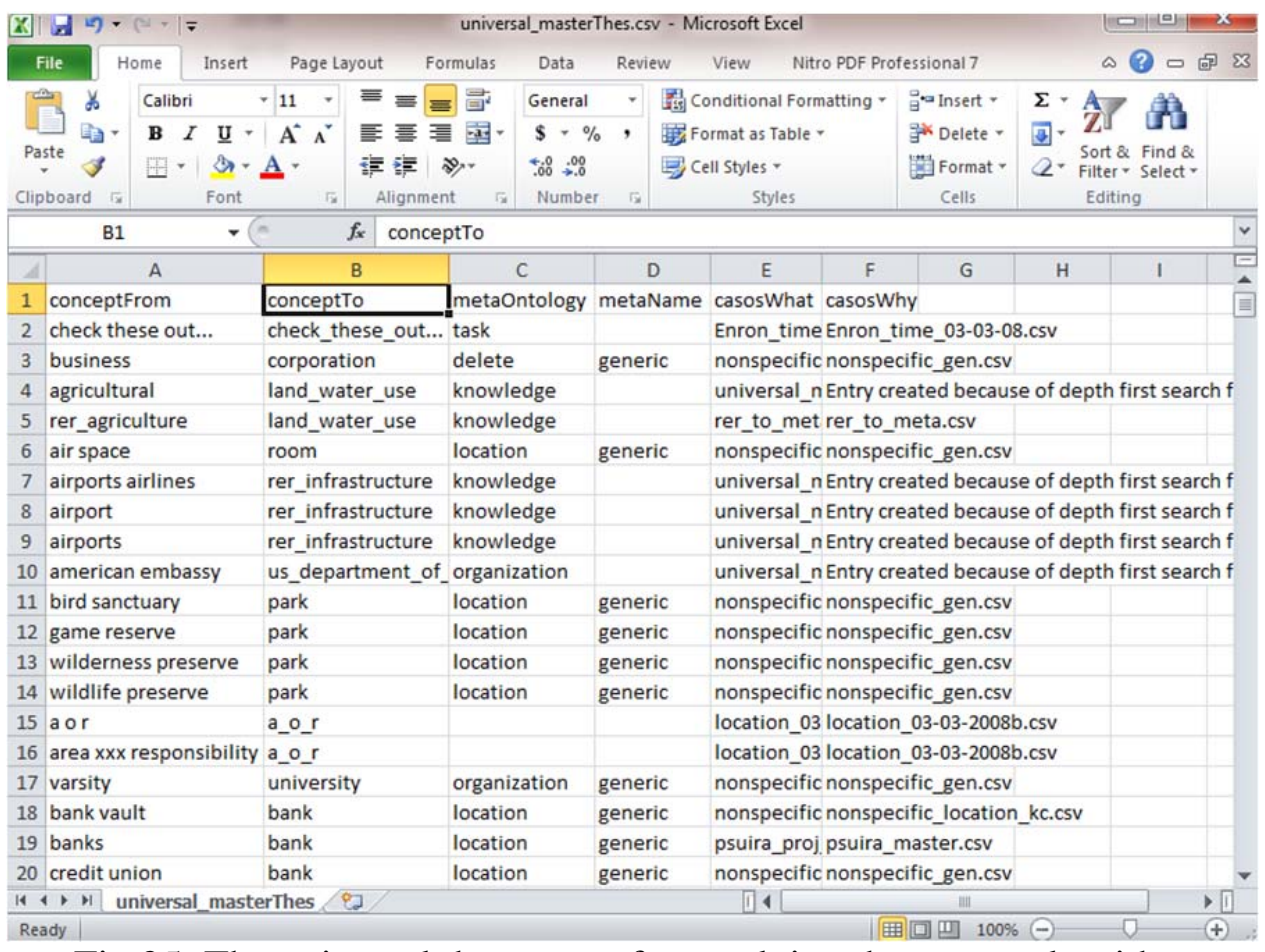

Fig 25: The universal thesaurus after applying the merge algorithm 


\section{Illustration}

After we created a universal thesaurus and the domain thesauri using the above described process, we analyzed the results and hence, the efficiency of this process by running the analysis on a sample data set. For this purpose, we used the Sudan data set.

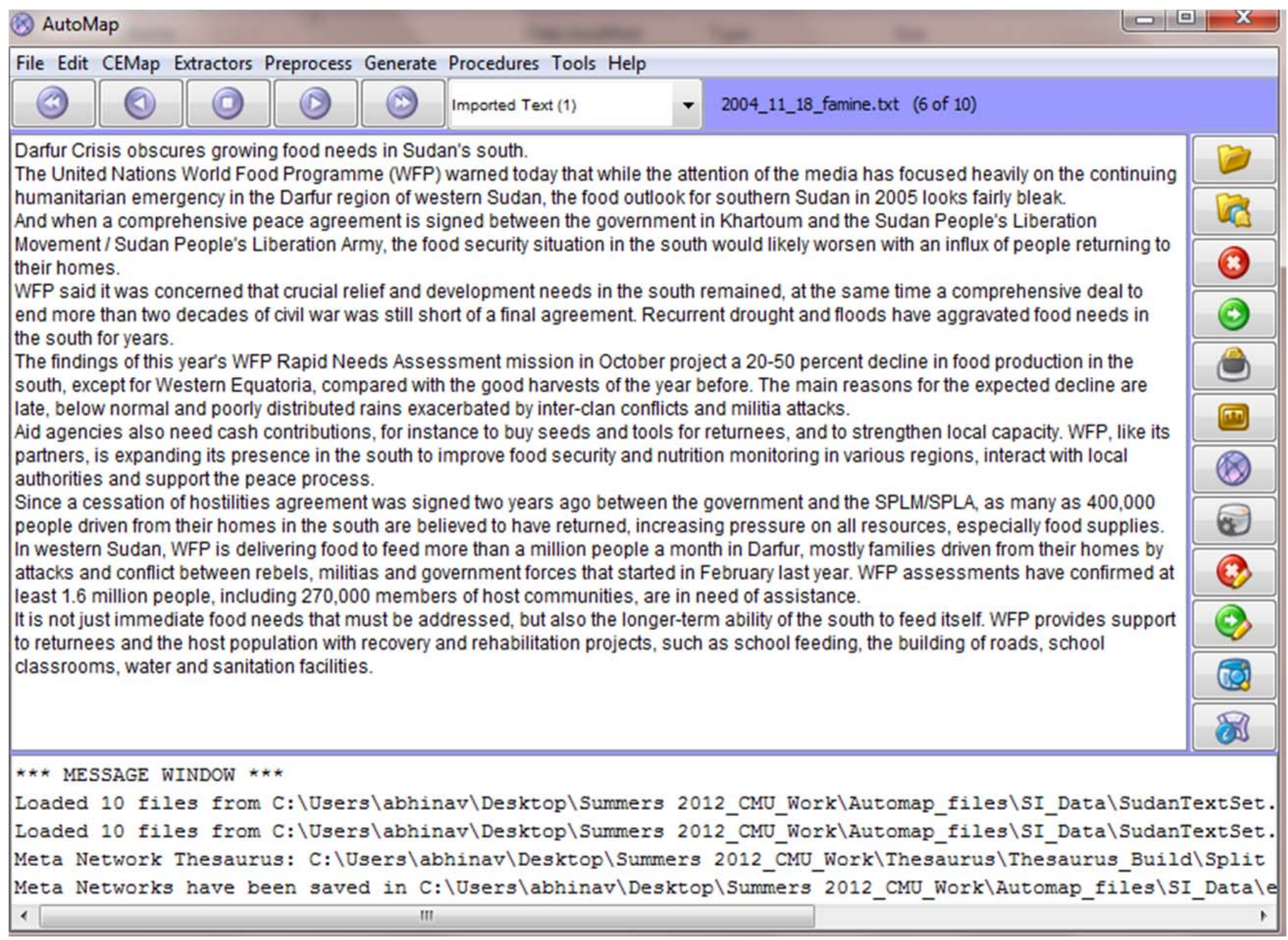

Fig 26: The Sudan data set. 
Firstly, we refine the raw Sudan data by applying the delete lists. The figure shown below is the data set after the universal and the domain delete lists are applied. Since we selected the rhetorical type of deletion; we see $\mathbf{x x x}$ in place of the deleted words. Note: This is the remainder data after applying both the universal and the Sudan domain delete lists.

Observations: The words which were present either in the universal delete list as noise words or the domain thesaurus as the non-important concepts have been marked as $\mathbf{x x x}$ in the figure below.

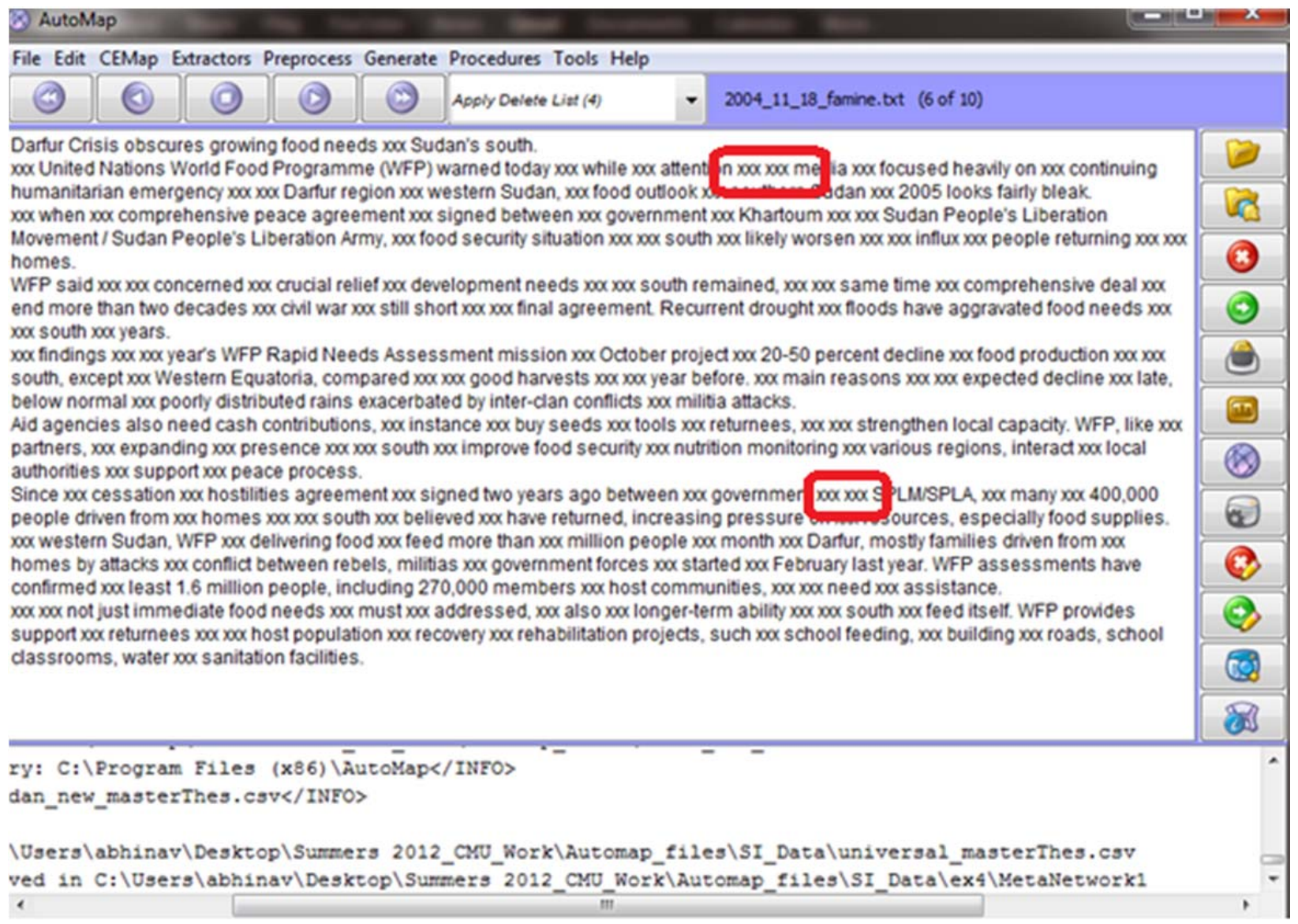

Fig 27: Remainder data after applying the delete lists. 
In order to further analyze the data and review the efficiency of our merge process, we created the meta network using the universal thesaurus as the standard thesaurus. The figure shown below shows the meta network when we load the dynetml file in ORA.

Observations: A meta network which consists of some concepts which have been classified according to their meta ontology mappings in the new universal master thesaurus.

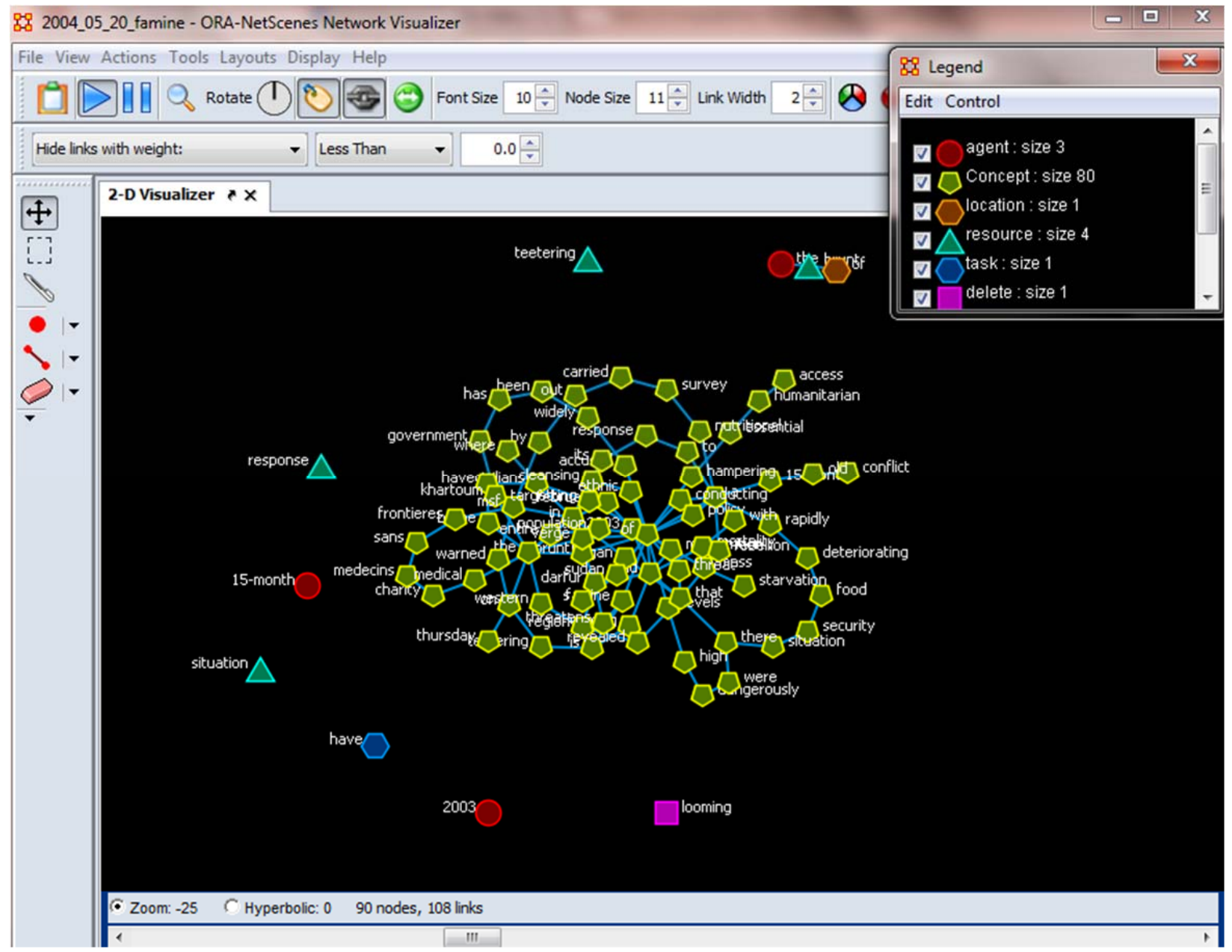

Fig 28: Meta network obtained after the universal thesaurus is applied. 
Next, we apply the universal delete list for the text refinement and afterwards we create a Dynetml file for the meta network using AutoMap and universal_masterThes.csv as the standard thesaurus.

Observations: The concept size has reduced by around $20 \%$ by applying the universal delete list before the Meta network generations. The number of concepts is 66 as compared to the previous observation of 80 . Also, we see a reduction in the number of agents by $33 \%$ after applying the universal delete list.

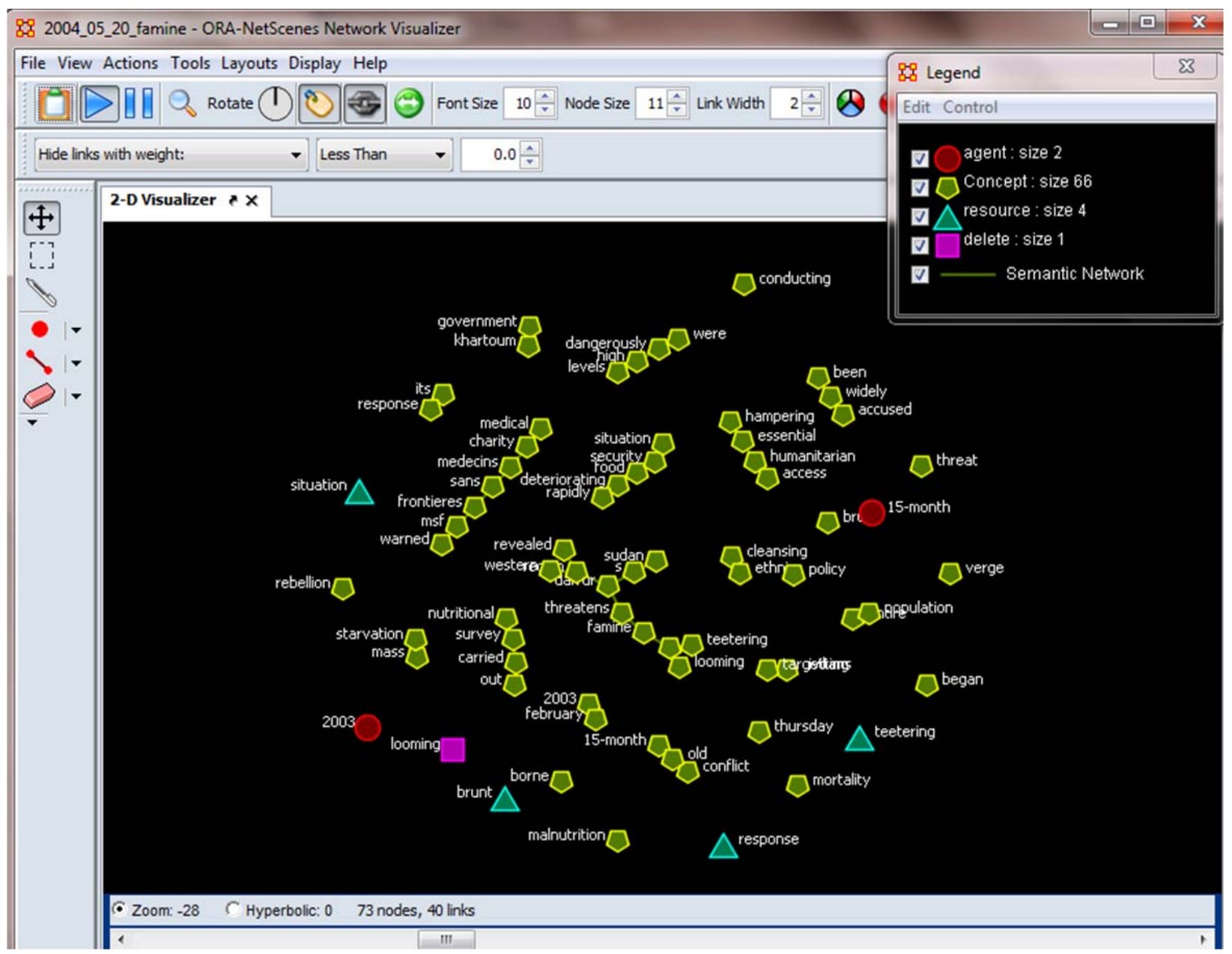

Fig 29: Meta network obtained after the universal thesaurus is applied along with the universal delete list 
The figure below shows the screenshot when the Dynetml file is loaded in ORA. This Dynetml file contains a Meta network which is created when we apply the domain delete list along with the universal_masterThes.csv used as the standard thesaurus.

Observations: A meta network is obtained in which some of the concepts have been classified according to the meta ontologies mapping in the universal master thesaurus. Others are identified as just concepts.

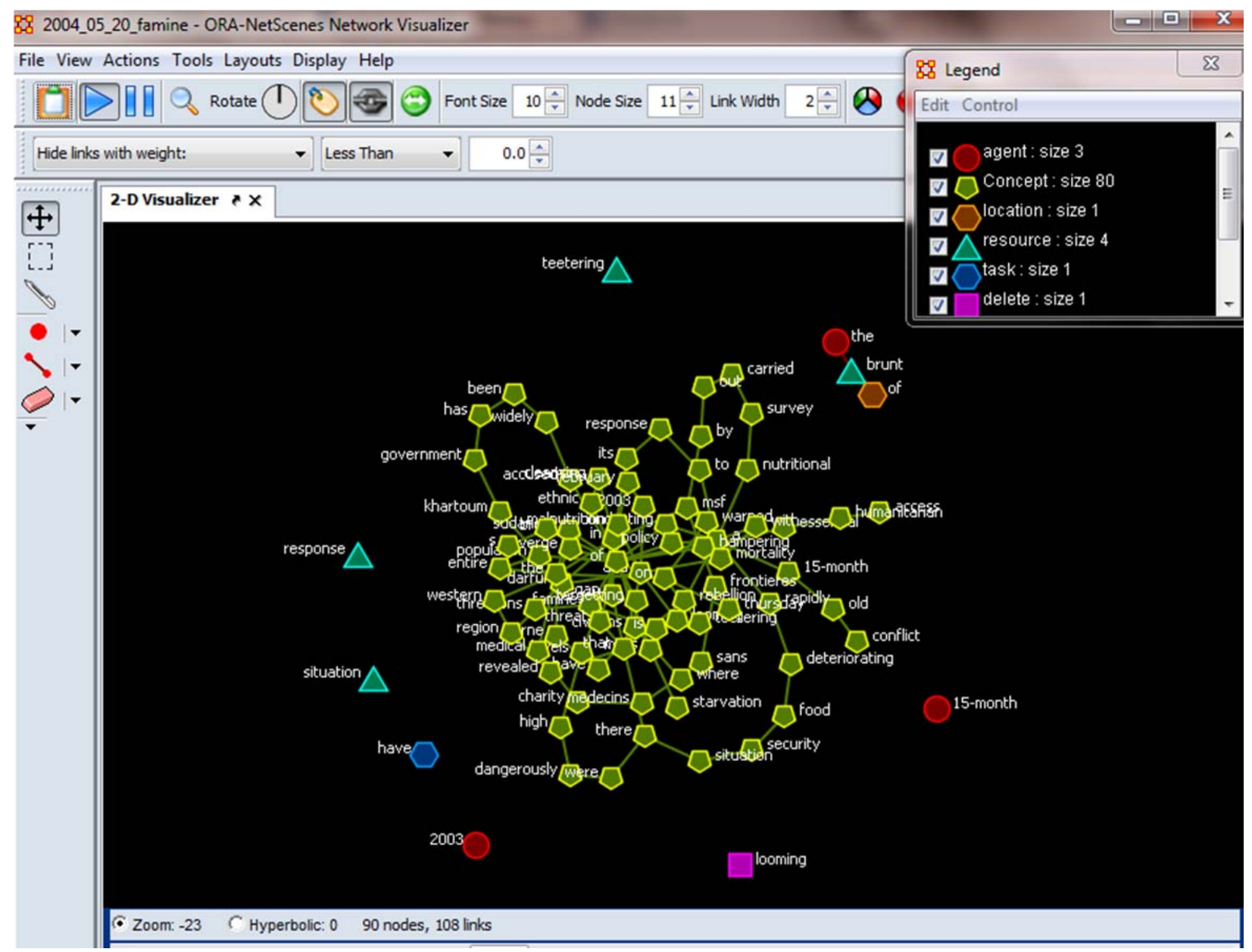

Fig 30: Meta network obtained after the domain delete list is applied along with the universal_masterThes.csv used as the standard thesaurus. 
Finally, we apply both the universal delete list and the domain delete list. After we have done the text refinement, we apply the domain thesauri as the generalization thesauri. Next, we create a Meta network using the universal_masterThes.csv used as a standard thesaurus.

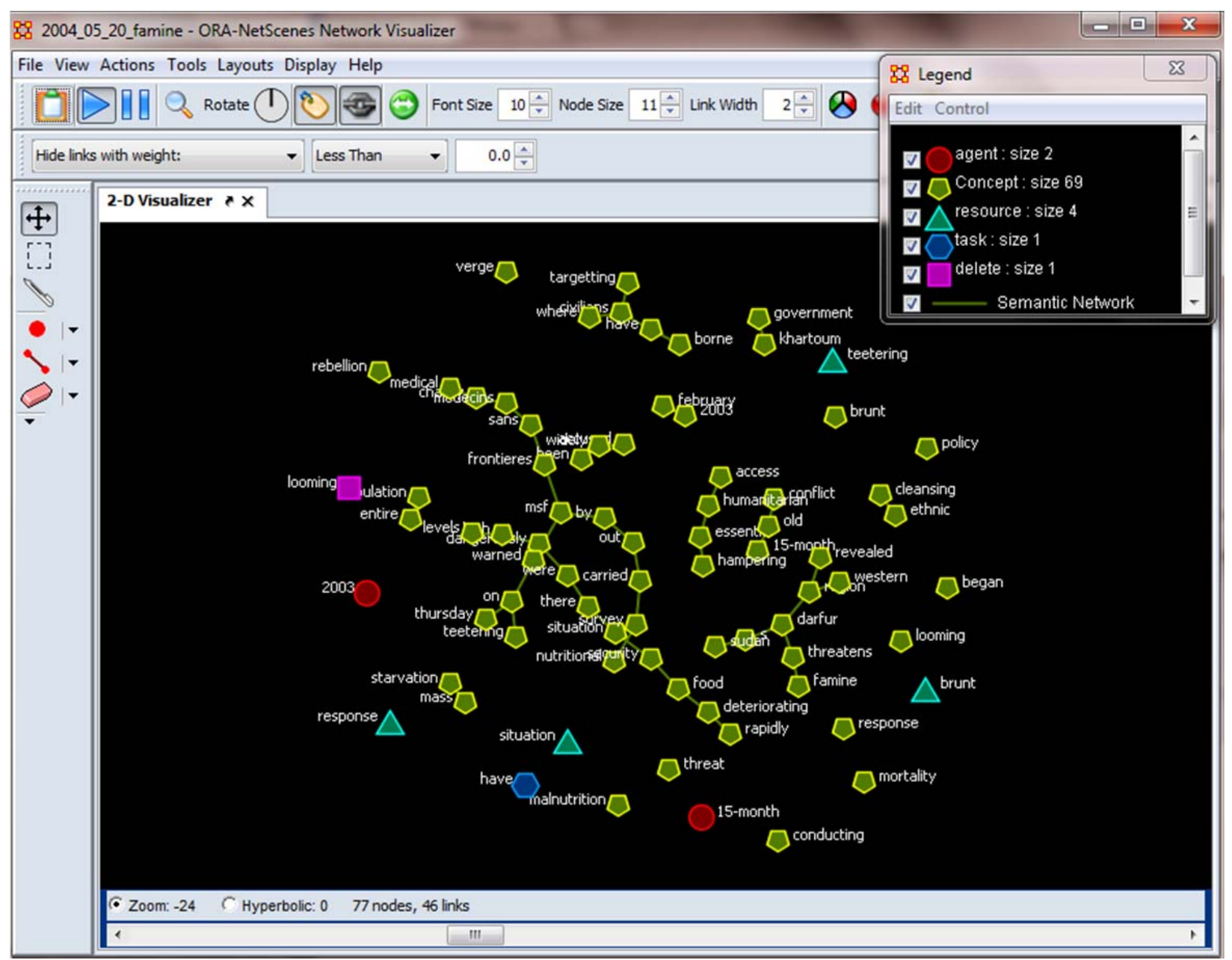

Fig 31: Meta network obtained by using both the universal and the domain delete lists, and using the domain thesauri and the universal thesaurus as the standard thesaurus. 


\section{Creating and Applying Change Files in ORA}

In this section, we discuss how to create the change files in ORA and apply the changes in ORA itself. The users can use this approach to modify the Meta networks extracted from the texts using AutoMap. By using this approach, the users can directly apply the changes to Meta network without using AutoMap for modifying the nodes, links and the networks.

\subsection{Creating Change files in ORA}

Step 1: Load the Meta network in ORA. The users can go to file->Open Meta-network and then browse to the location of the required network files or just simply drag and drop the dynetml file

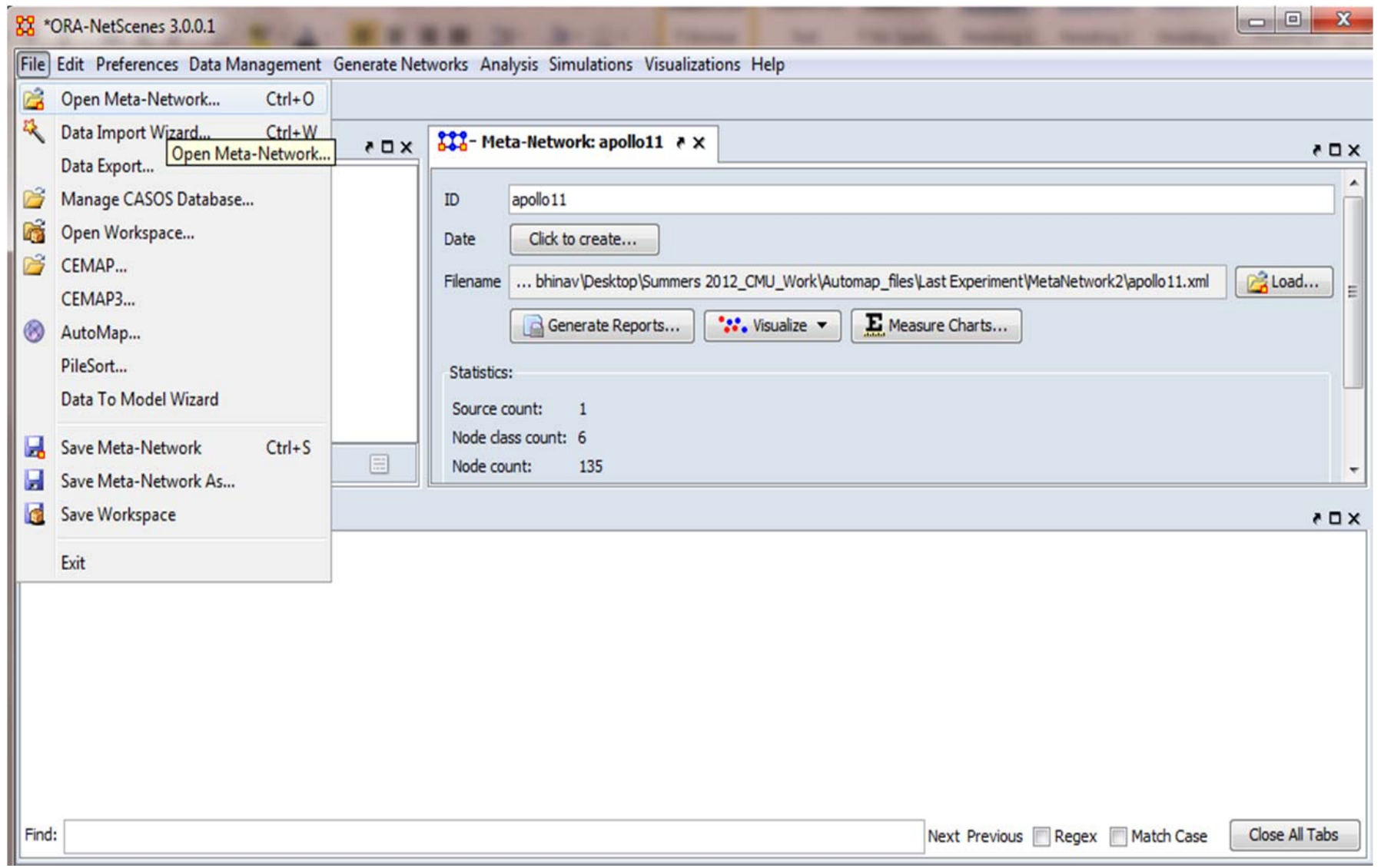

Fig 32: Opening Meta network in ORA. 
Step 2: Once the Meta network is opened in ORA, select the node class in which you want to make any changes. If the node class is uncertain to the users, they can simply just click on the node class "concepts", which includes the concepts from all the node classes. After this, the user should go to the "editor" tab.

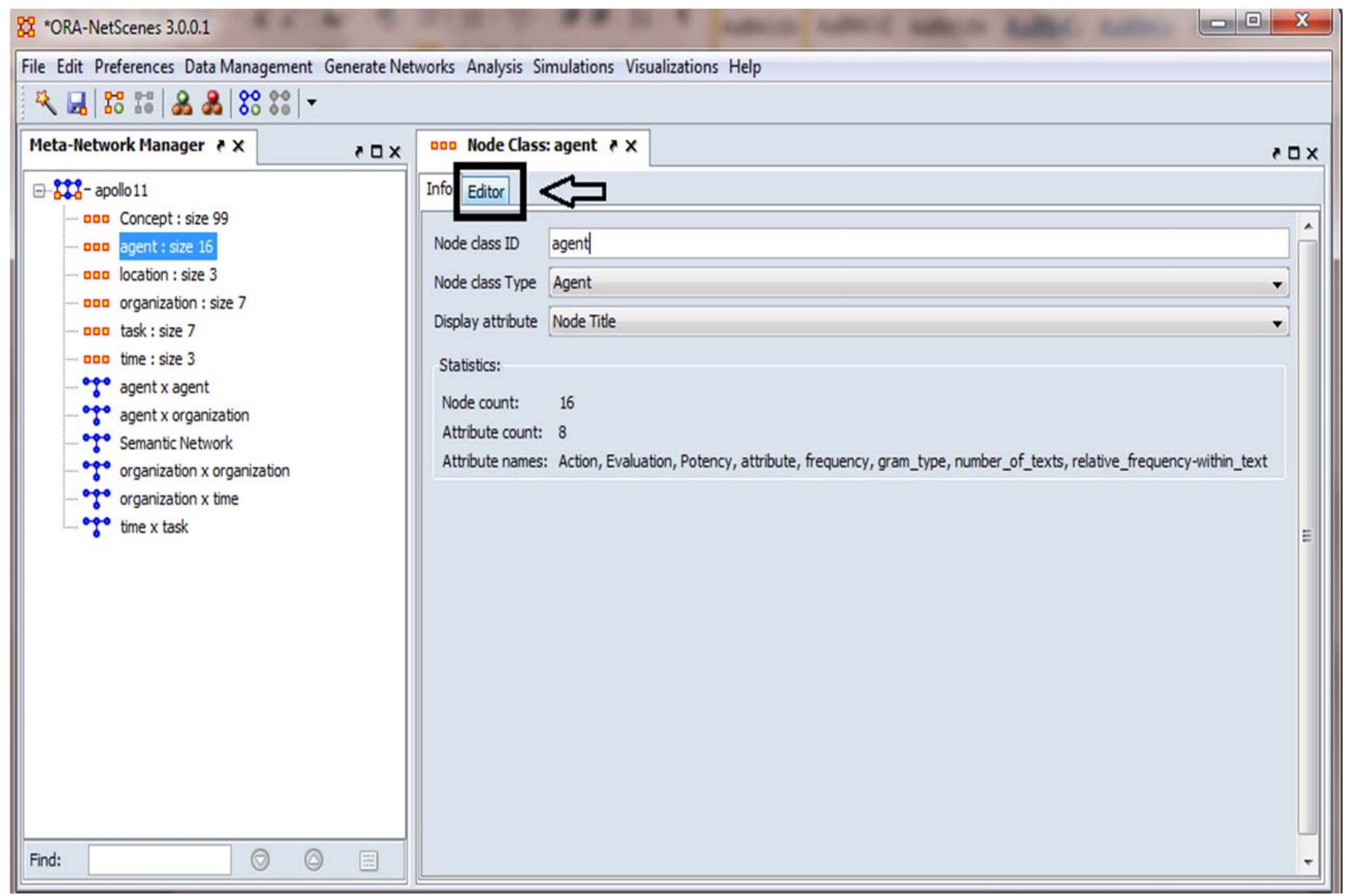

Fig 33: Modifying the nodes in ORA. 
Step 3: Once the editor tab is opened corresponding to the node class where the required node class changes are to be made, the user should select the node on which the changes are to made. When the node is selected, a check appears on the left and the row is highlighted in yellow. After this, the users can select from a number of node class operations. These operations are Delete node, moving the node to a different node class, copying the node to a different node class etc. For example: we want to delete the agent "buzz". We select the node buzz and delete this node from the network.

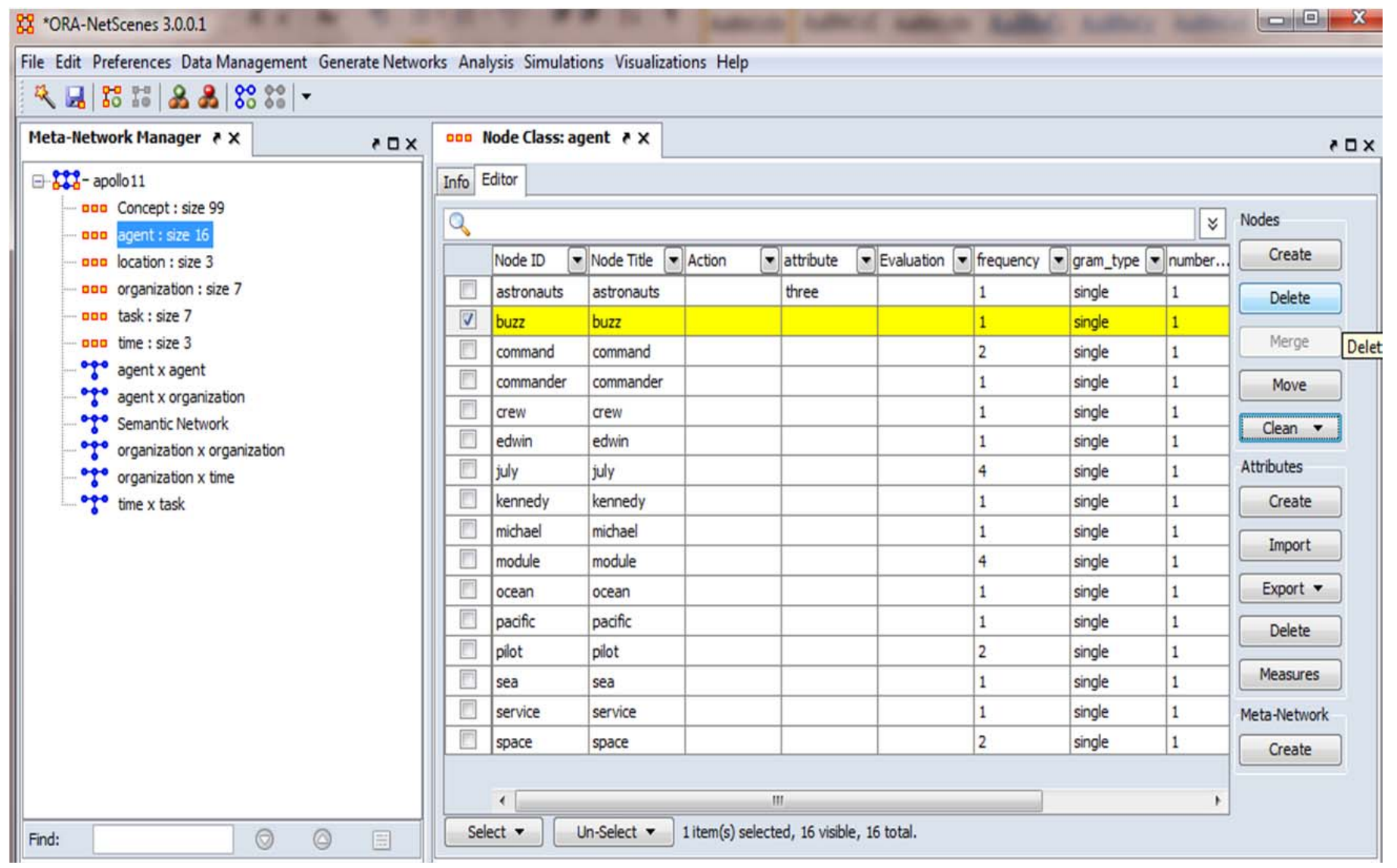

Fig 34: Deleting a node in ORA. 
Step 4: After the nodes are modified in the Meta network, we are ready to save this change list. Saving this change list helps us to use this list for future reference and modifying network afterwards. The users should go to Data Management-> Meta network clean. A window pops out and then the user should select the required Meta network wherein the node changes were made.

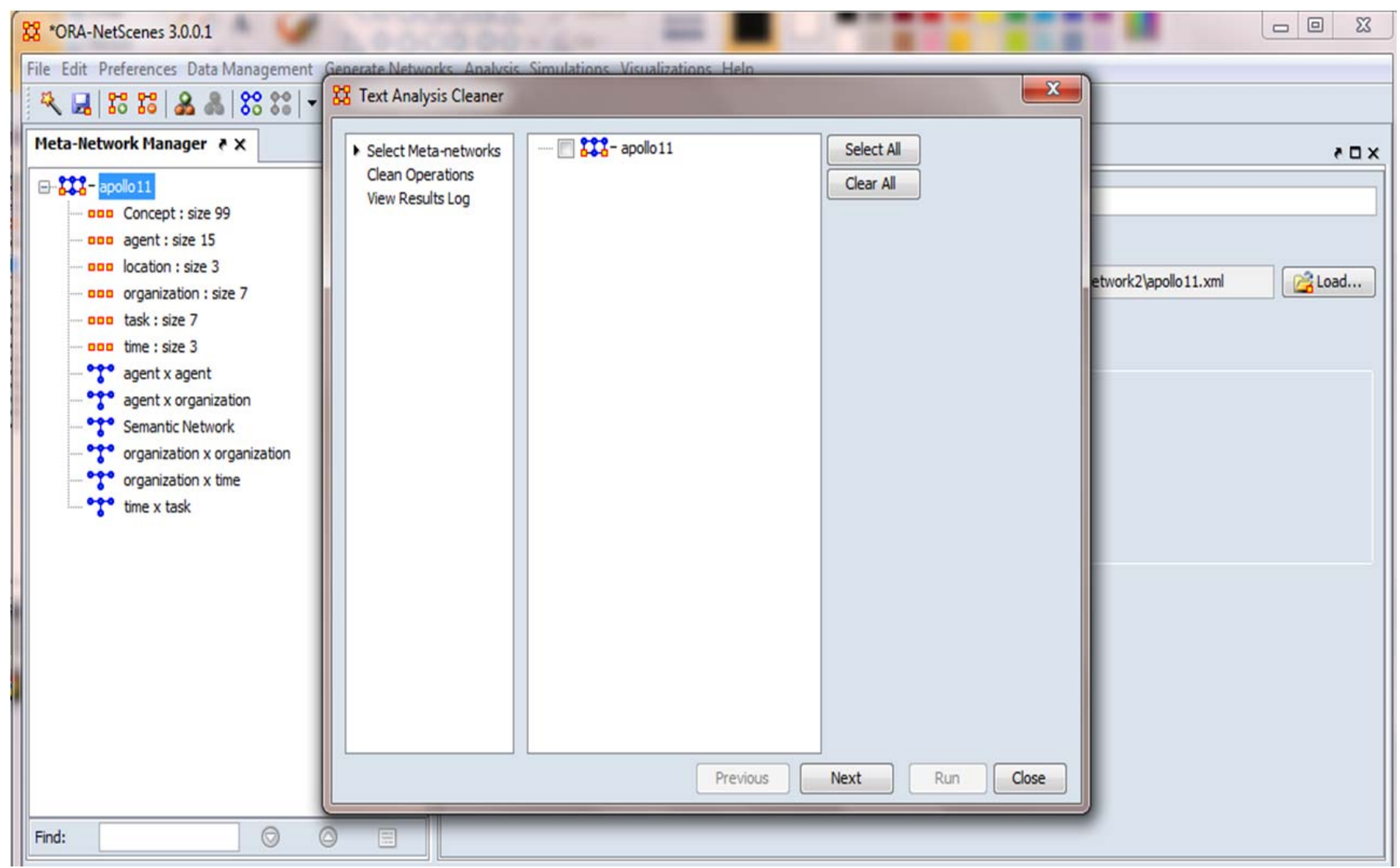

Fig 35: Creating a change list in ORA. 
Step 5: Select the Meta network and then click next. In the clean operations menu, select the tab create change list. The users need to provide a base name for the changed file and then select the node classes for which the changed file is required. Then click RUN. After the process is completed, click Close.

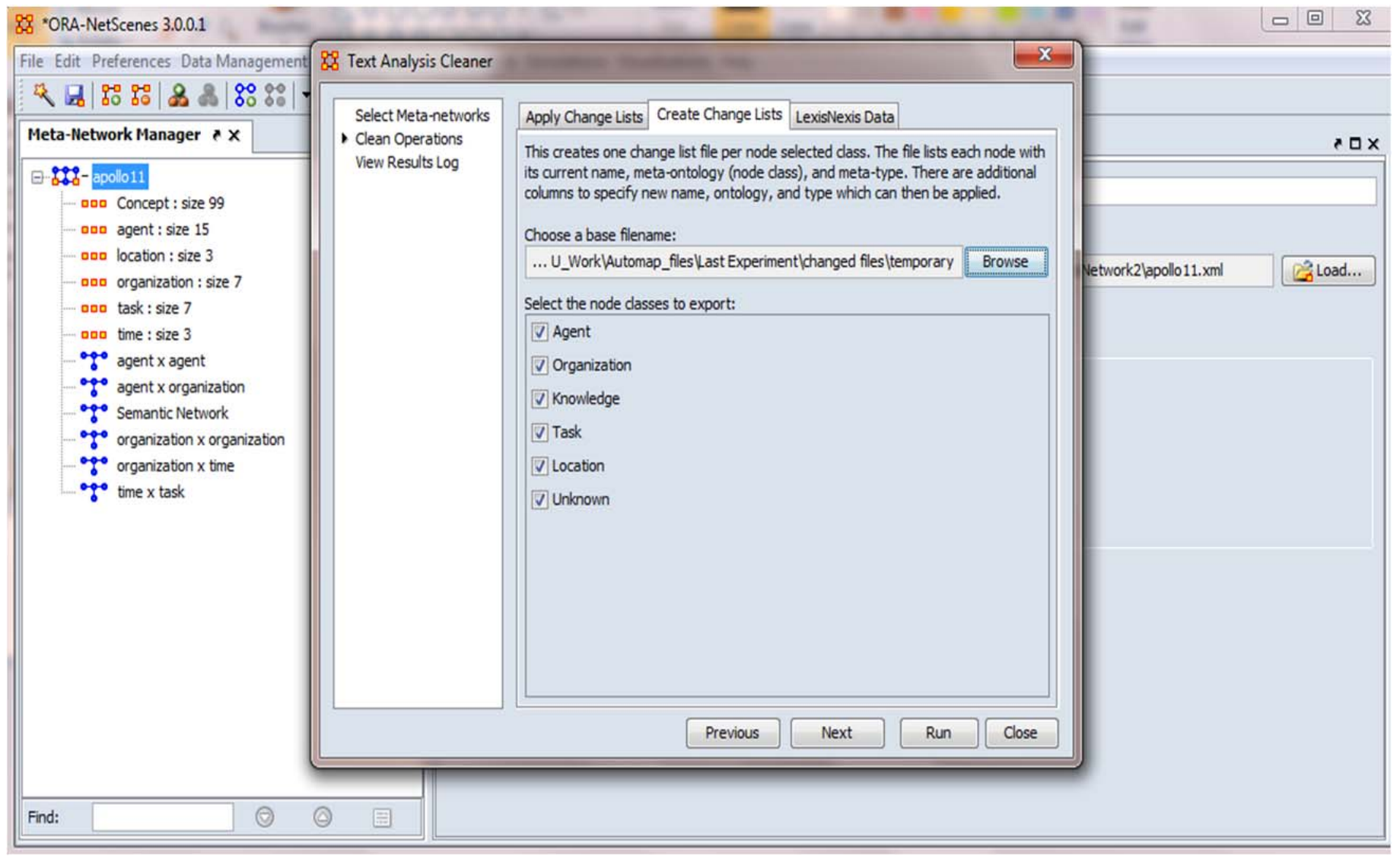

Fig 36: Saving a change list in ORA. 
Step 6: Browse to the location of the change files and explore the change files. We see that the change files resemble the reduced format ( See section 3.5 on page).

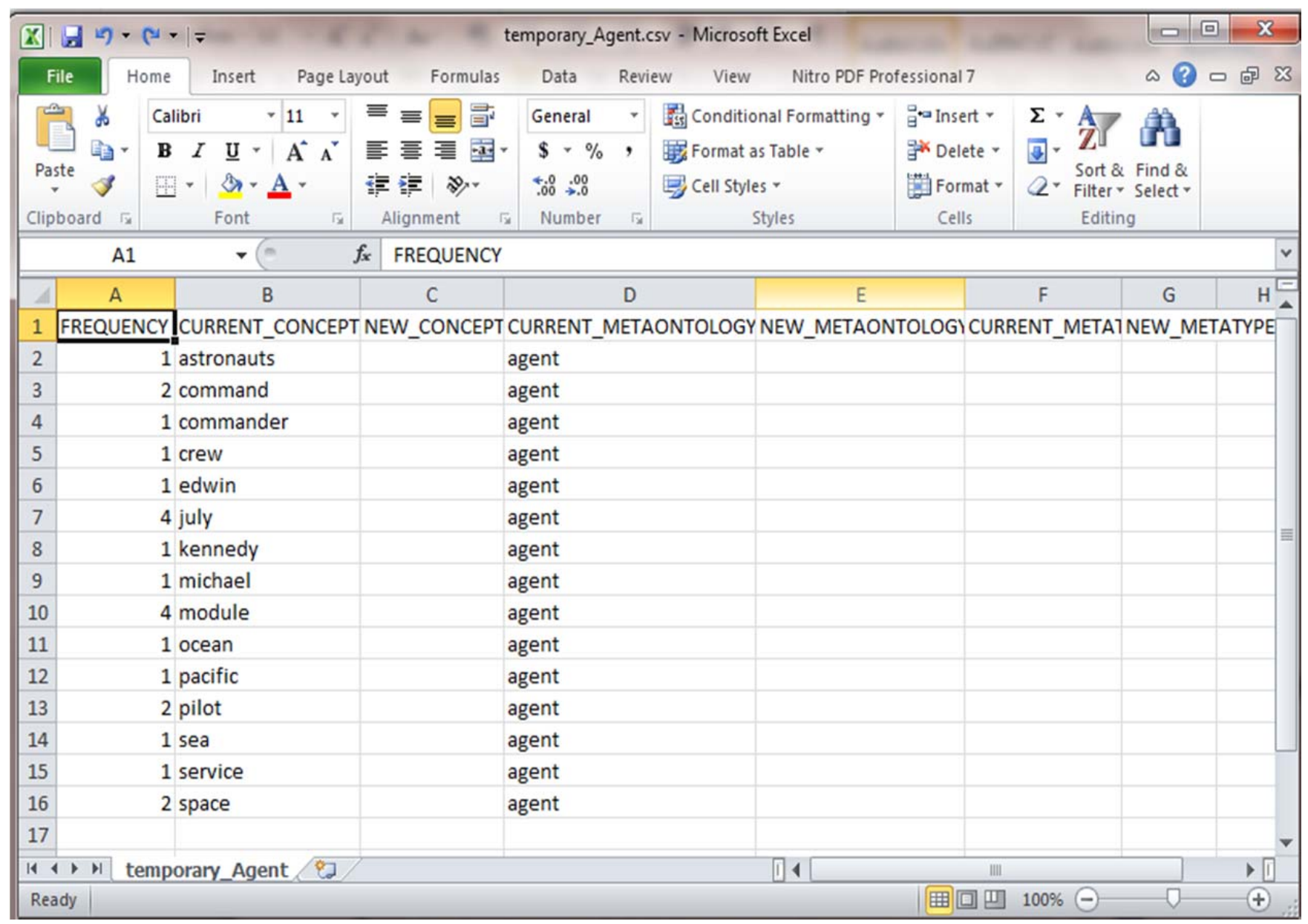

Fig 37: The format for the change file. 


\subsection{Applying change files in ORA}

We can also apply the change files in ORA to update the Meta networks. We will describe a step-by-step approach to apply the change files in ORA.

Step 1: Load the Meta network in ORA. The users can go to file->Open Meta-network and then browse to the location of the required network files or just simply drag and drop the dynetml file

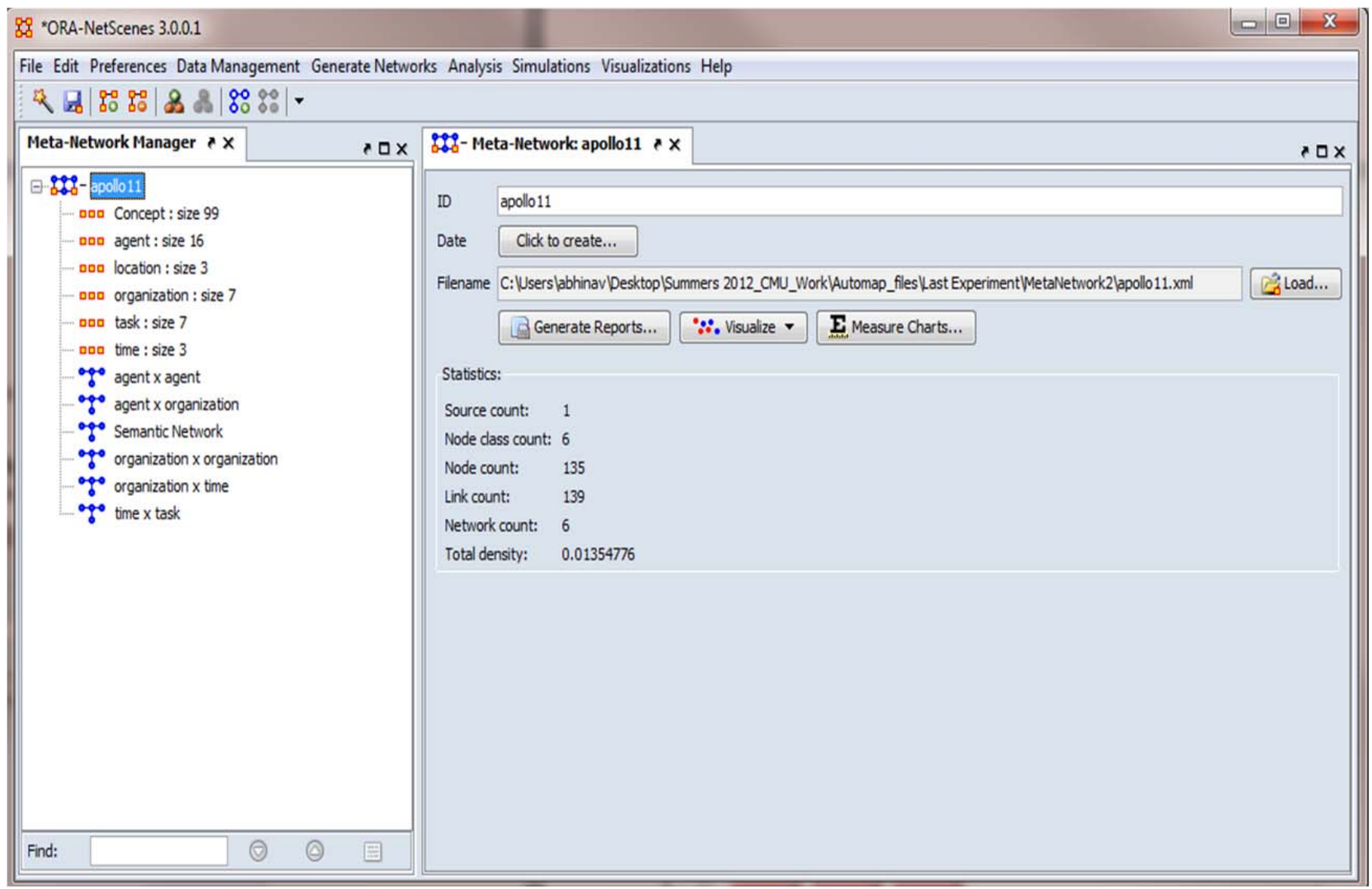

Fig 38: Meta network opened in ORA 
Step 2: The users should go to Data Management-> Meta network clean. A window pops out and then the user should select the required Meta network wherein the node changes are to be made.

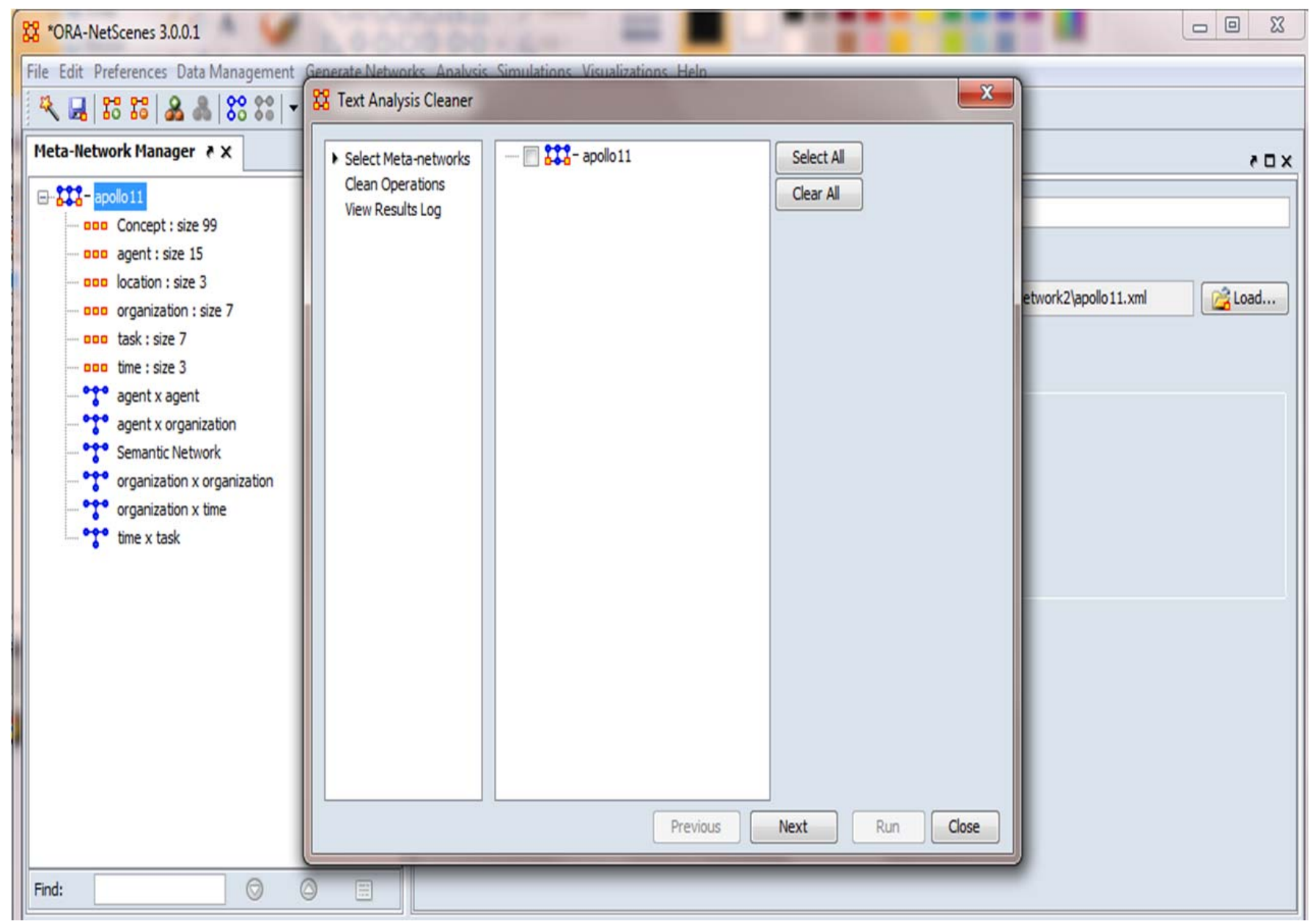

Fig 39: Selecting the Meta network where the changes are to be applied. 
Step 3: Select the Meta network and then click next. In the clean operations menu, select the tab Apply change list. The users need to provide the location for the changed list file and then select all the node classes for which the changed file in which the changes are required. Then click RUN. After the process is completed, click Close.

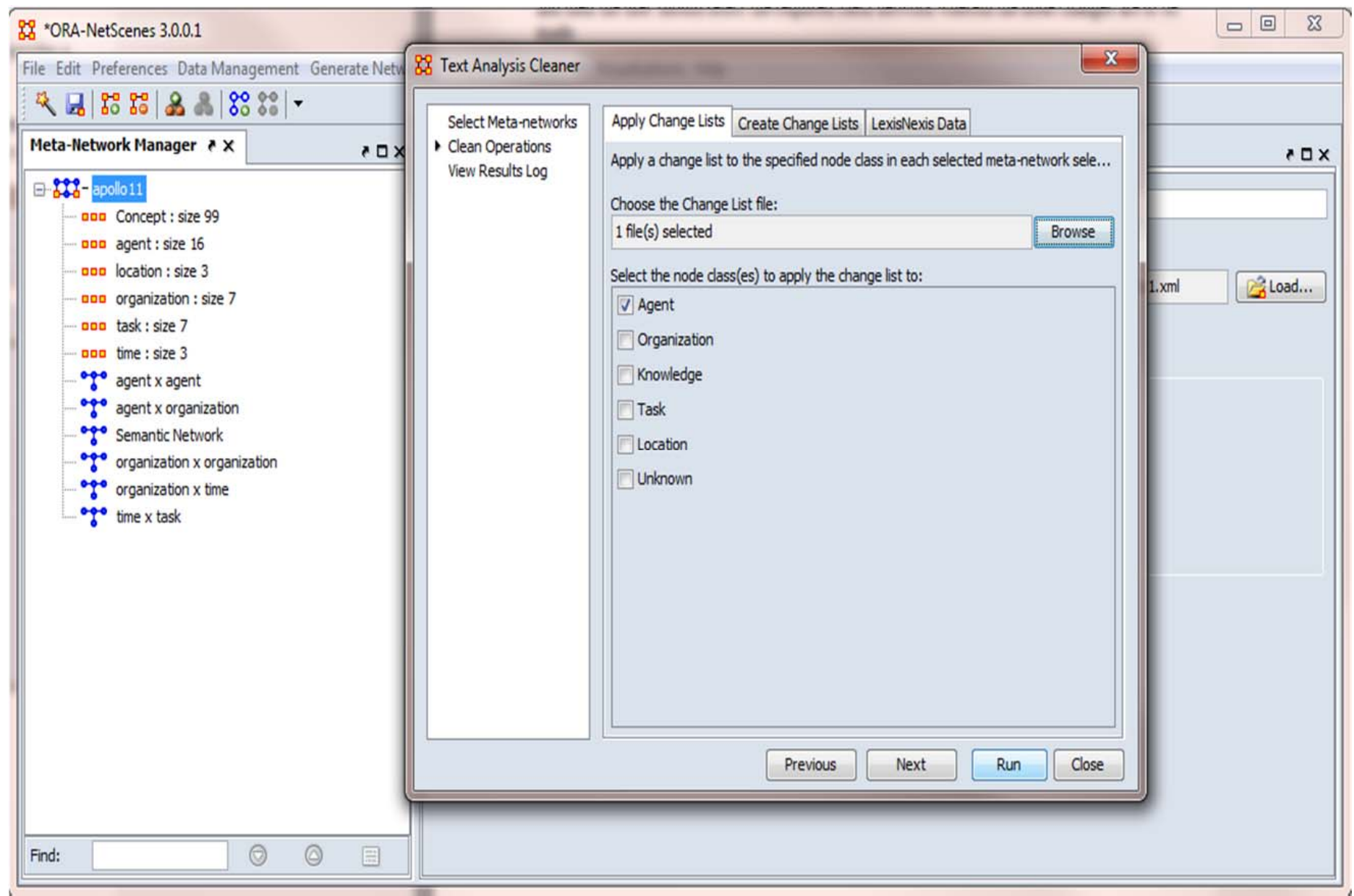

Fig 40: Applying the change list in ORA. 


\section{Results}

After we successfully created a universal thesaurus and several other domain thesauri for various projects (such as Enron, Catnet, Sudan etc.), in order to analyze the efficiency of the merge and split process, we compared the results of the Catnet data set using the standard thesaurus (i.e., the old version of the universal merged thesaurus) and the new Universal merged thesaurus created with the process described in this report.

The data set we used to compare the results of the D2M process [3] using the standard thesaurus and the merged thesaurus were related to the interviews that took place with one of the members of the Al-mujaharoon group. To get a better idea of the difference of the results between the outputs of the D2M run [3] using the standard and the merged thesaurus, we generated the key entity reports [2] for the two D2M [3] outputs.

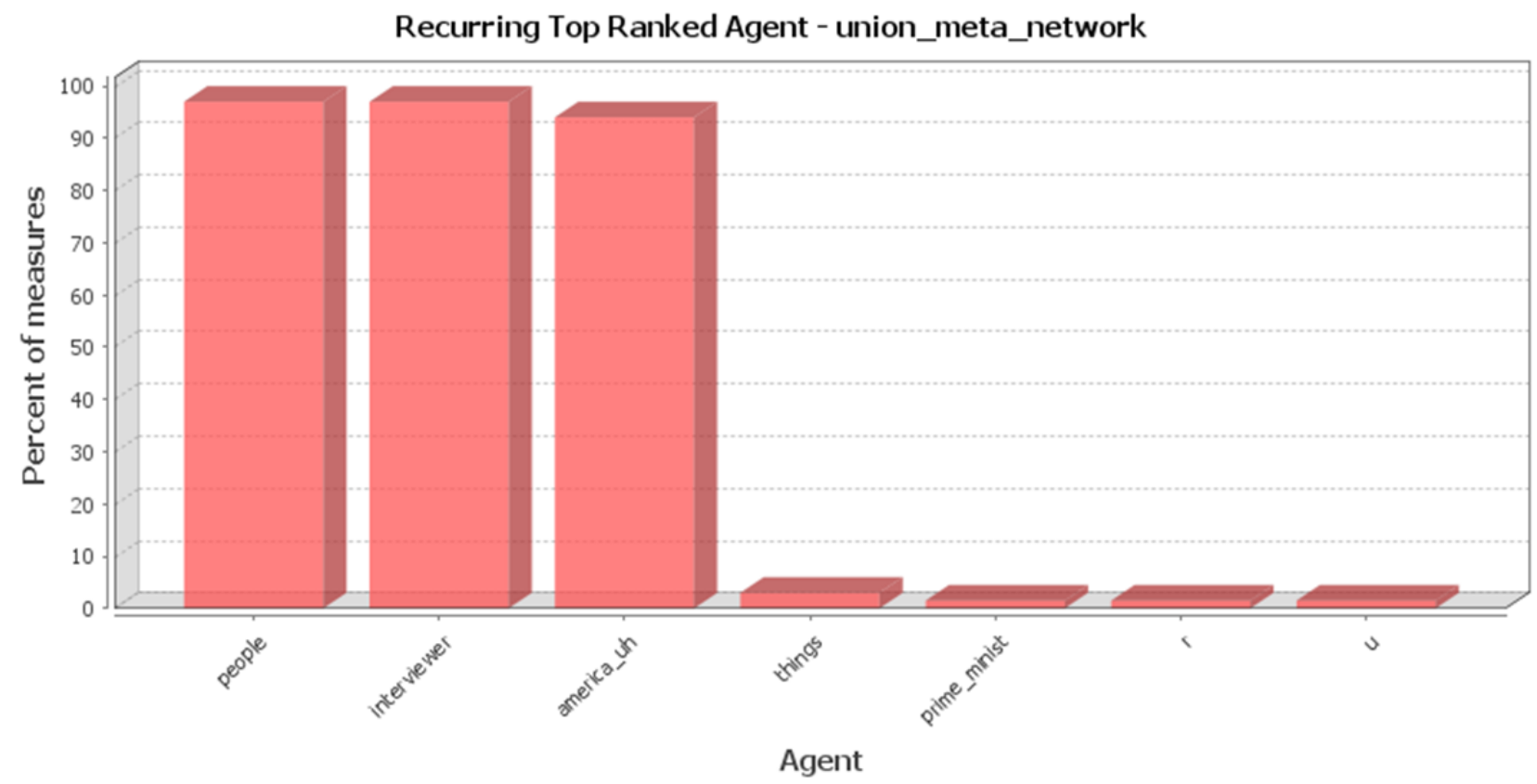

Fig 41: Top ranked agents using the Standard thesaurus 


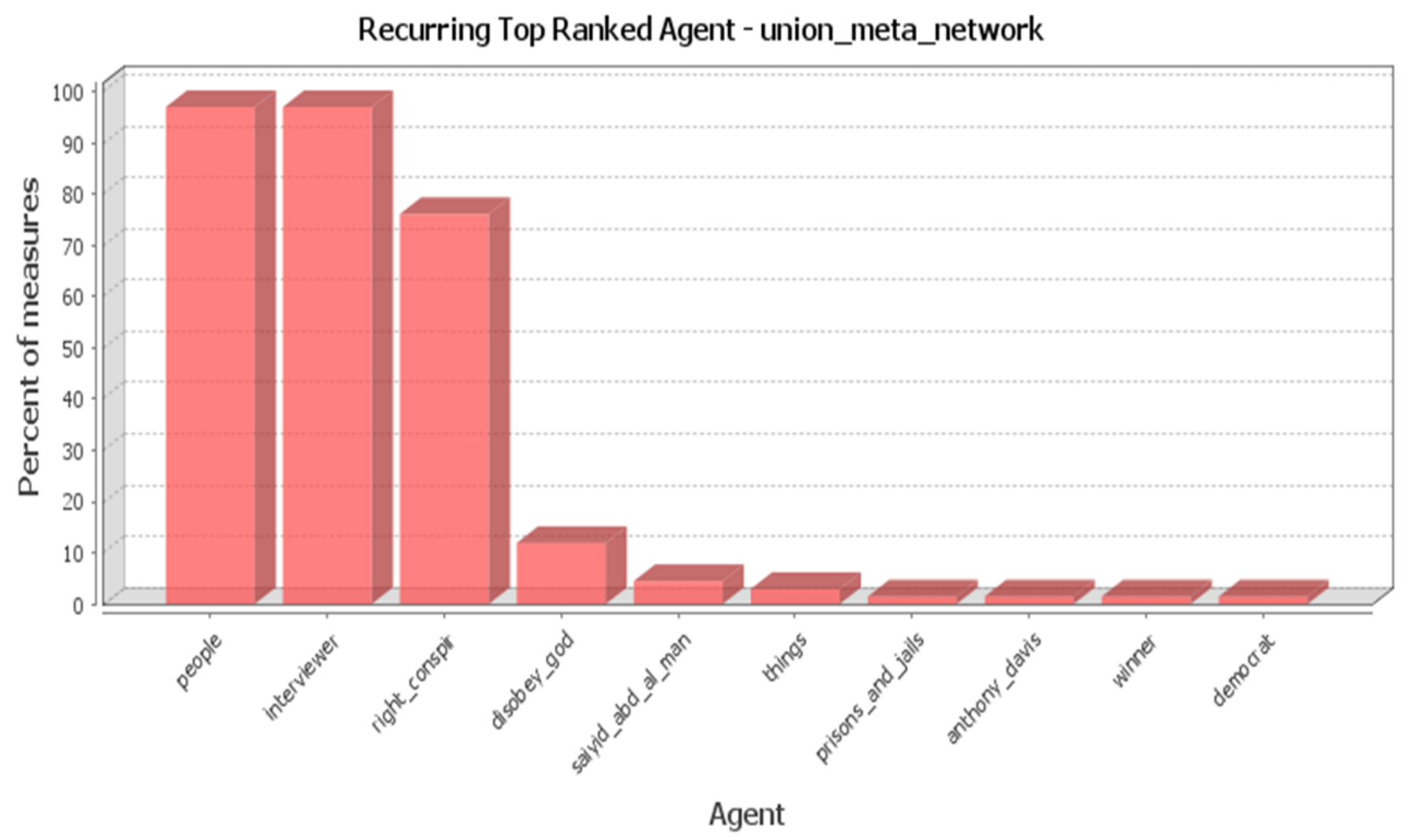

Fig 42: Top ranked agents using the Merged Thesaurus

The above two figures depict the recurring top ranked agents for the D2M run using the standard thesauri and the D2M run using the merged thesauri. The bar graph depicts the top 10 recurring agents in the 24 measures used for a qualitative analysis in the key report.

The 24 measures are as follows:

1. Emergent Leader (cognitive demand)

2. In-the-Know (total degree centrality)

3. Number of Cliques (clique count)

4. Most Knowledge (row degree centrality)

5. Most Resources (row degree centrality)

6. Leader of Strong Clique (eigenvector centrality)

7. Leader of Strong Clique Per Component (eigenvector centrality per component)

8. Acts as a Hub (hub centrality)

9. Acts as an Authority (authority centrality)

10. Potentially Influential (between ness centrality)

11. Potentially Influential Links (edge between ness centrality)

12. Connects Groups (high between ness and low degree)

13. Specialization - knowledge (relatively unique)

14. Complete Exclusivity - knowledge (complete exclusivity)

15. Specialization - resource (relatively unique) 
16. Complete Exclusivity - resource (complete exclusivity)

17. Specialization - task (relatively unique)

18. Complete Exclusivity - task (complete exclusivity)

19. Specialization - event (relatively unique)

20. Complete Exclusivity - event (complete exclusivity)

21. Specialization - location (relatively unique)

22. Complete Exclusivity - location (complete exclusivity)

23. Workload (actual based on knowledge and resource)

24. Group Awareness (shared situation awareness)

It was observed that the top 10 recurring agents in the standard D2M run included agents, $\mathbf{r}$ and u which were completely irrelevant when considering the key agents. Items such as these indicate errors in the standard thesauri.

In contrast, the observations with regard to the top 10 key agents emergent in the key entity report using the merged thesaurus run were more relevant and informative. The new key entity report was able to do away with erroneous entities in the top 10 agents like " $r$ " and " $\mathrm{u}$ ".

Secondly, agents like “saiyid_abd_al_man” and "Anthony Davis” which emerged as two of the top 10 ranked agents using the merged thesaurus were not even present in the key entity report generated using the standard thesaurus.

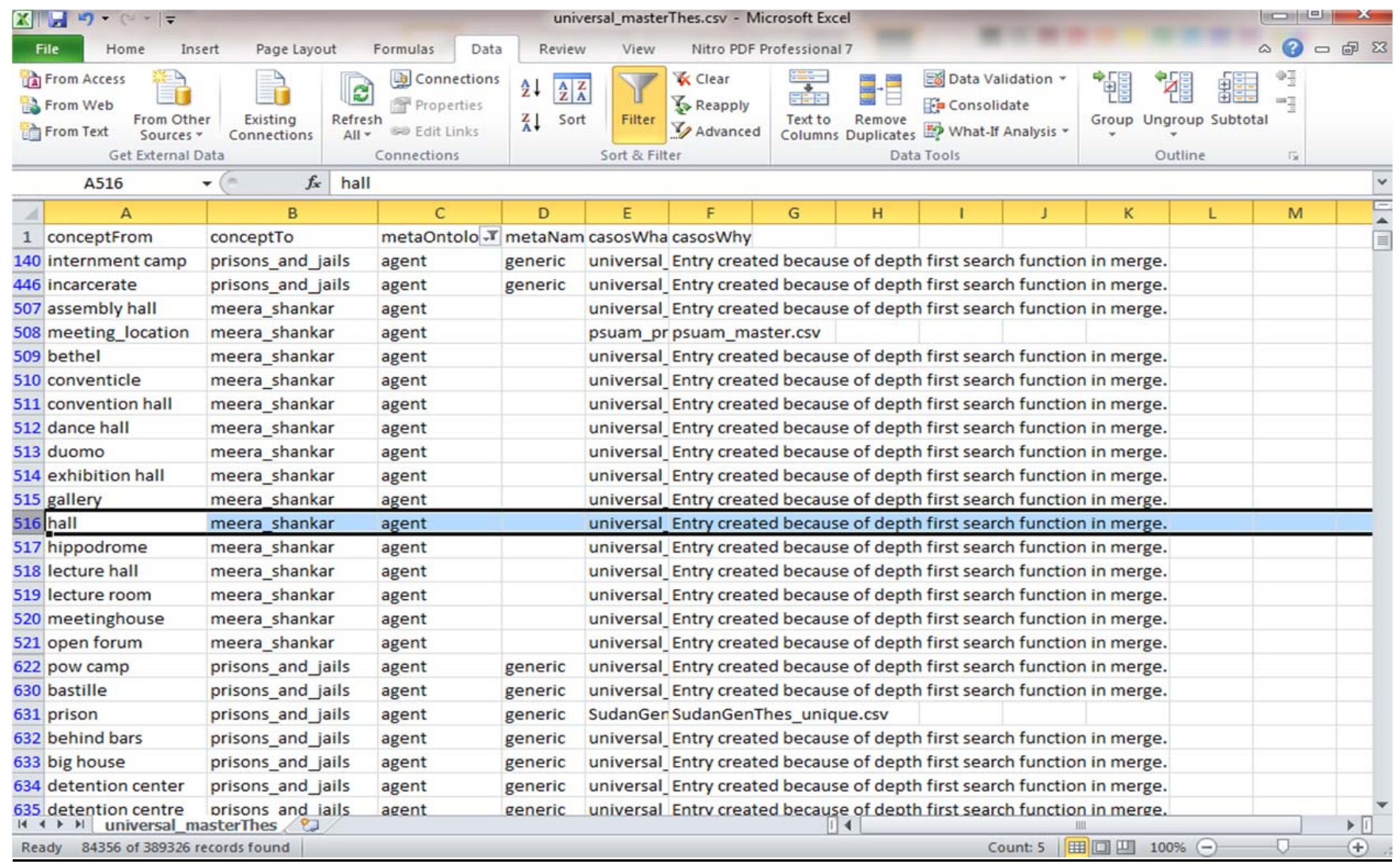

Fig 43: Universal_masterThes.csv 


\section{Future Directions}

In the near future, we would want this process to get more automated so as to reduce the human effort involved in combining all the files in a single directory and manually listing the files in the splitcontrol.csv and mergecontrol.csv.

One of the major problems which exist in the existing process is that the files in the sub directory will not be read from the input directory. This means that if we want to keep some files separate and to merge as well, this would be difficult and cumbersome.

The existing process takes the input files in a particular format, i.e. the master thesaurus format. If the user provides the merge routine with a file in any other format, this file would not get merged. So, one of the improvements could be to automatically convert all the input files into the required format and then do the merge step.

As stated, the merge and the split process takes the files as input in master thesaurus format which is less informative then the reduced format type (which is a 7 column type thesaurus). So, another improvement with regard to the formats would be to support input files in the change format so that the output in turn becomes more informative and much more descriptive.

We would also like to use the change files created from ORA directly in AutoMap, so that gives the users a method to manipulate the changes in AutoMap and incorporate them in ORA and vice-versa.

Additional drawbacks of this merge and split process involve the use of depth first search in the merge procedure. This means that if a concept like hall is general it might get linked to something like meera_shankar because of the function depth first search. This in turn might create some shocking and unexpected results in the key entity reports [2] and hence, the overall analysis. 


\section{References}

[1] Carley, Kathleen \& Columbus, Dave \& Azoulay, Ariel (2012). AutoMap User's Guide 2012 Carnegie Mellon University, School of Computer Science, Institute for Software Research, Technical Report CMU-ISR-12-106.

[2] Carley, Kathleen \& Columbus, Dave (2012). Basic Lessons in ORA and AutoMap 2012 Carnegie Mellon University, School of Computer Science, Institute for Software Research, Technical Report CMU-ISR-12-107.

[3] Carley, Kathleen \& Bigrigg, Michael \& Diallo, Bouba. Data-to-Model: A Mixed Initiative Approach for Rapid Ethnographic Assessment. Carnegie Mellon University, School of Computer Science, Institute for Software Research, Technical Report. 